\title{
A long-term study of the impulsive choices of Lewis and Fischer 344 rats
}

\author{
Carlos F. Aparicio ${ }^{1}$ • Mirari Elcoro ${ }^{2}$ - Benigno Alonso-Alvarez ${ }^{3}$
}

Published online: 11 April 2015

(C) Psychonomic Society, Inc. 2015

\begin{abstract}
Contemporary analyses of choice were implemented to analyze the acquisition and maintenance of response allocation in Lewis (LEW) and Fischer 344 (F344) rats. A concurrent-chains procedure varied the delay to the larger reinforcer $(0.1,5,10,20,40$, and $80 \mathrm{~s})$. Delays were presented within sessions in ascending, descending, and random orders. Each condition lasted 105 days, and the entire data set was analyzed to obtain discounting functions for each block of 15 sessions and each food delivery across delay components. Both a hyperbolic-decay model and the generalized matching law described well the choices of LEW and F344 rats. Estimates of discounting rate and sensitivity to the immediacy of reinforcement correlated positively. The slope of the discounting function changed with presentation orders of the delays to the larger reinforcer. Extended training reduced differences between the LEW and F344 rats in discounting rates, sensitivity to the immediacy of reinforcement, and estimates of the area under the curve. We concluded that impulsive choice can change as a function of learning and is not a static property of behavior that is mainly determined by genetic and neurochemical mechanisms. Choosing impulsively may be an advantage for organisms searching for food in rapidly changing environments.
\end{abstract}

Carlos F. Aparicio

caparicio@salemstate.edu

1 Department of Psychology, Salem State University, 352 Lafayette Street, Salem, MA 01970-5353, USA

2 Department of Psychology, Armstrong State University, Savannah, GA, USA

3 Department of Psychology, University of Oviedo, Oviedo, Spain
Keywords Acquisition · Delay discounting · Fischer 344 . Impulsive choice $\cdot$ Lewis $\cdot$ Maintenance $\cdot$ Rats

Impulsive choice has been defined as choosing a smaller immediate reinforcer over a larger but delayed reinforcer (Ainslie, 1974; Rachlin \& Green, 1972), and delay discounting is the process by which the efficacy (value) of the larger reinforcer decreases with increasing delay to its delivery (Myerson \& Green, 1995). In choice situations in which the smaller immediate reinforcer (the smaller-sooner reinforcer, or SSR) and the larger delayed reinforcer (the larger-later reinforcer, or LLR) are concurrently available, choosing the LLR is an instance of self-controlled choice (Logue, 1988), and choosing the SSR is a case of impulsive choice (Mazur, 2000). The hyperbolic-decay model describes the degree to which the value of the LLR decays with increasing delay (Mazur, 1987):

$V=\frac{A}{1+k D}$

where $V$ is the reinforcer value, $A$ is reinforcer amount, $D$ is the reinforcer delay, and $k$ is a free parameter estimating how fast the value of the LLR decays with increasing $D$.

The accuracy of the hyperbolic-decay model in describing the data from numerous studies with human (e.g., Myerson \& Green, 1995; Rachlin, Raineri, \& Cross, 1991) and nonhuman (e.g., Aparicio, Hughes, \& Pitts, 2013; Farrar, Kieres, Hausknecht, de Wit, \& Richards, 2003; Green, Myerson, Shah, Estle, \& Holt, 2007; Mazur, 2012; Stein, Pinkston, Brewer, Francisco, \& Madden, 2012) animals is remarkably general, and this model does so with a single free parameter $(k)$. Studies of impulsivity using changes in $k$ to assess the effects of drugs or other neurobiological variables on impulsive choice have often used the adjusting-delay (i.e., Mazur 
1987) and the adjusting-amount titration procedures (i.e., Richards, Mitchell, de Wit, \& Seiden, 1997; Green et al., 2007), or the method developed by Evenden and Ryan (1996). One important distinction between these procedures is that the former generate graded discounting functions between sessions (e.g., Stein et al., 2012), and the latter generates an entire delay-of-reinforcement function within each session (e.g., Evenden \& Ryan, 1999); these methods serve different purposes when examining variables affecting impulsive choice. All of the procedures, however, share the following characteristics: (1) Discrete trials offer a choice between the SSR and the LLR; (2) forced-choice trials precede free-choice trials, exposing subjects to the contingencies associated with the SSR and the LLR; (3) forced- and free-choice trials require a single response to produce either the SSR or the LLR; and (4) an intertrial interval follows each SSR and LLR, keeping constant the time between choices. Some advantages and disadvantages of each of these procedures in generating graded discount functions have been reviewed (Madden \& Johnson, 2010), warranting other procedures to examine impulsive choice.

In the present study, we used a concurrent-chains procedure, recently developed to study impulsive choice in rats (Aparicio et al., 2013). In this technique, the delay to deliver the LLR is manipulated dynamically within sessions, capitalizing on Evenden and Ryan's (1996) procedure to obtain an entire delay-of-reinforcement function within each session. Choice is estimated by analyzing the distribution of responses on two levers concurrently available in the initial link (e.g., Grace, 1994), preventing the development of an exclusive preference for one or the other alternative, which is observed when only one response is required to choose and produce the SSR and the LLR (Mazur, 1987, 2010). Another characteristic of the present procedure is that the presentation order of delays to deliver the LLR was manipulated across conditions (i.e., ascending, descending, or random presentation orders), avoiding the possibility of creating a single history of increasing delay to deliver the LLR; this characteristic is important because, in studies in which discrete trials have been used to create a history of increasing delay to LLR delivery, choice for the LLR decreased across blocks of trials during probe sessions in which the delay to LLR delivery remained at $0 \mathrm{~s}$ throughout the session (e.g., Evenden \& Ryan, 1996; Pitts \& McKinney, 2005; Slezak \& Anderson, 2009). In addition, the present procedure required locomotion; rats traveled from the front to the back wall of the chamber to press a lever to restart each cycle of the concurrent-chains procedure, which implied effort, representing some cost to reach the choice point. This is important because effort plays a crucial role in preference (Salamone \& Correa, 2009), determining sensitivity to relative changes in the frequency, amount, and immediacy of reinforcement (e.g., Aparicio, 2001; Aparicio \& Cabrera, 2001).
The primary goal of the present study was to examine the impulsive choices of Lewis (LEW) and Fischer 344 (F344) rats, analyzing and representing the entire data set instead of using the data from the final sessions of each condition. The adjustments in the concurrent-chains procedure developed to study impulsive choice (i.e., Aparicio et al., 2013) and the use of contemporary measures to analyze impulsivity are the contributions of the present study. One objective was to investigate the acquisition and maintenance of impulsive choice, studying changes in discounting functions and sensitivity to the immediacy of reinforcement as a function of training (Aparicio et al., 2013). Another objective was to analyze the role of presentation orders of the delays to LLR in determining the slope of the discounting function; this objective is important to ratify the findings of studies with human (e.g., Robles \& Vargas, 2007, 2008; Robles, Vargas, \& Bejarano, 2009) and nonhuman (e.g., Fox, Hand, \& Reilly, 2008; Slezak \& Anderson, 2009) animals showing that the slope of the discounting function is affected by the order in which delays to LLR are presented in the choice situation. The last objective was to compare the impulsive choices of LEWs and F344s, given the growing interest of the scientific community in using these rat strains to assess the effects of drugs and neurobiological variables in determining the slope of the delaydiscounting function; this objective is significant because researchers have suggested that genetic and neurochemical differences between LEWs and F344s account for the dissimilarities in impulsive choice between these inbred rats (e.g., Anderson \& Diller, 2010; Garcia-Lecumberri et al., 2011).

Evidence suggesting genetic differences between LEWs and F344s has come from models of dependence on alcohol (Suzuki, George, \& Meisch, 1988), nicotine (Brower, Fu, Matta, \& Sharp, 2002), cocaine (Kosten et al., 1997), etonitazene (Suzuki, George, \& Meisch, 1992), and morphine (Martin et al., 2003), showing that self-administration of these substances occurred more readily in the former than in the latter strain of rats. This cumulative body of data indicates that LEWs possess a phenotype highly susceptible to drug addictions (Garcia-Lecumberri et al., 2011), providing a genetic model of human drug abuse (Kosten \& Ambrosio, 2002). Neurochemical variations between LEWs and F344s were documented in parametric analyses of neurotransmitter systems such as the serotonergic (Chaouloff, Kulikov, Sarrieau, Castanon, \& Mormede, 1995), dopaminergic (Flores, Wood, Barbeau, Quiron, \& Srivastava, 1998; Lindley, Bengoechea, Wong, \& Schatzberg, 1999), and noradrenergic (Sziraki et al., 2001) systems. Studies comparing and contrasting the dopamine (DA) and serotonin (5-HT) systems of LEWs with those of F344s have reported that the former strain has lower levels of DA and 5-HT in many brain areas (Burnet, Mefford, Smith, Gold, \& Sternberg, 1996), fewer $\mathrm{D}_{2}$ receptors in the striatum and nucleus accumbens core, fewer $\mathrm{D}_{3}$ receptors in the nucleus accumbens shell and olfactory tubercule (Flores et al., 
1998), and fewer 5-HT binding sites in the hippocampus and frontal cortex (Selim \& Bradberry, 1996) than F344s. The LEWs also display higher levels of basal N-methyl-Daspartate receptors in many regions of the brain and reduced proenkephalin mRNA levels in the dorsal striatum and nucleus accumbens than do F344s (Martin et al., 1999, Martin et al., 2003).

Behavioral and physiological differences between LEWs and F344s have been reported in various laboratories (see Kosten \& Ambrosio, 2002). For instance, studies using an autoshaping technique (Brown \& Jenkins, 1968) to establish lever pressing for food found that the LEWs acquired that behavior more rapidly and performed it at higher rates than the F344s (Kearns, Gomez-Serrano, Weiss, \& Riley, 2006); similar results were documented by Anderson and Elcoro (2007) using a tandem fixed-ratio one, fixed-time 20 s schedule of reinforcement to establish lever pressing for food. Moreover, studies of impulsive choice in LEWs and F344s have shown steeper discounting functions for the former than for the latter strain of rats (e.g., Anderson \& Diller, 2010; Anderson \& Woolverton, 2005; Huskinson, Krebs, \& Anderson, 2012; Madden, Smith, Brewer, Pinkston, \& Johnson, 2008). Nonetheless, the generality of this finding has been compromised by research that failed to show differences in discounting functions between LEWs and F344s (e.g., Stein et al., 2012; Wilhelm \& Mitchell, 2009).

The discrepancies in the discounting functions of the LEWs and F344s are probably due to methodological differences in the ways to obtain graded discounting functions. Studies using steady-state procedures, arranging a delay/ amount combination for at least ten sessions before replacing it for a different combination, found steeper discount functions in LEWs than in F344s (e.g., Anderson \& Diller, 2010; Anderson \& Woolverton, 2005; Huskinson et al., 2012; Madden et al., 2008). In contrast, studies that have used a rapid-determination adjusting-amount procedure to control each delay/amount combination in series of five sessions have yielded no differences in discounting functions between LEWs and F344s (Stein et al., 2012; Wilhelm \& Mitchell, 2009), suggesting that more than five sessions are required for the rapid-determination adjusting-amount procedure to detect a strain difference that is mainly driven by the LEWs. Perhaps the F344s need several sessions (ten or more) to detect rapid changes in the contingencies of reinforcement and to show steady-state performance. If so, initial differences in discounting rates and higher values of $k$ for the LEWs than for the F344s (estimated by Eq. 1) should appear after ten or more sessions, showing that measures of delay discounting can change systematically as a function of training. A recent study by Aparicio et al. (2013) provided a positive answer to this question by using a concurrent-chains procedure. In that study, the performance of LEWs and F344s was compared at different points of training (i.e., blocks of 15 days each) under conditions of extended exposure (i.e., 225 sessions) that varied the delay to the LLR in the terminal links of a concurrentchains procedure. Early in training (Blocks 1-2), there were no differences between the LEWs and the F344s, with both strains showing flat discounting functions. In Blocks 4 and 5, the LEWs showed more sensitivity to the effects of delay to the LLR (i.e., steeper discounting functions) than did the F344s; this difference in discounting functions between strains remained for ten blocks of sessions. However, it faded by Blocks 14 and 15, with results showing no differences between the LEWs and F344s in proportions of LL choices, discounting rates ( $k$ in Eq. 1), and other dependent variables derived from the general form of the matching law (i.e., Baum, 1974; Baum \& Rachlin, 1969). It was concluded that (1) measures of delay discounting change systematically as a function of training, (2) the F344s require more training than the LEWs to detect dynamic changes in the contingencies of reinforcement, and (3) with extended training, the F344s develop patterns of impulsive choice that are similar (i.e., comparable $k$ s) to those observed in the LEWs (Aparicio et al., 2013).

Although in the Aparicio et al.'s (2013) study the proportion of LL choices was reasonably high (i.e., .8) when $5 \mathrm{~s}$ was the minimal delay to obtain either the SSR or the LLR, a $0-\mathrm{s}$ delay to deliver these reinforcers was not included in their choice procedure. Another potential drawback of Aparicio et al.'s study is that fixed-interval (FI) schedules were used in the terminal links to delay the LLR, requiring one response at the end of the interval to obtain the reinforcer (i.e., compromising the effect of delay to LLR on choice); this might explain the low estimates of $k$ found in Aparicio et al.'s study.

In the present study, we corrected the above drawbacks by (1) including a delay close to $0 \mathrm{~s}(0.1 \mathrm{~s})$ to deliver both the SSR and the LLR, and (2) using fixed-time (FT) schedules in the terminal links to manipulate delays to the LLR, eliminating the response requirement at the end of the interval. The general goal was to use a range of measures (described in detail below) analyzing impulsive choice, and the specific objectives were to (1) examine the acquisition and maintenance of impulsive behavior, (2) analyze whether the presentation orders of the delays to the LLR is important in determining the slope of the discounting function, and (3) contribute to the study of the impulsive choices of LEWs and F344s.

\section{Method}

\section{Subjects}

Experimentally naïve inbred LEW $(n=8)$ and F344 $(n=8)$ male rats (Harlan Sprague-Dawley, Indianapolis, IN), between 108 and 122 days old at the start of training, served as the 
subjects. An ad-libitum feeding regimen of Purina Lab Chow was used at first to allow habituation to the laboratory. On the day before training, the feeders of all cages were emptied and the rats were placed on a regimen of food restriction; postsession feedings of approximately $10 \mathrm{~g}$ of Purina Lab Chow were provided, such that the weights of the LEWs and F344s at the beginning of the study ranged from 253 to $282 \mathrm{~g}$ and from 209 to $234 \mathrm{~g}$, respectively, and at the end of it, their weights ranged from 409 to $459 \mathrm{~g}$ and from 324 to $380 \mathrm{~g}$, respectively. Between sessions, the animals were individually housed in plastic cages with water permanently available in a temperature-controlled colony room providing a 12:12-h light/dark cycle (lights on at 0600).

\section{Apparatus}

Eight modular chambers (Coulbourn E10-11R TC) for rats measuring $295 \mathrm{~mm}$ long, $250 \mathrm{~mm}$ wide, and $285 \mathrm{~mm}$ high (inside) were enclosed in isolation cubicles (E10-23) that from the outside measured $794 \mathrm{~mm}$ long, $533 \mathrm{~mm}$ wide, and $514 \mathrm{~mm}$ high. The front and back walls of each chamber were made of stainless steel, and the sidewalls of Plexiglas. The floor of each chamber was a square metal grid (E10-18NS). A food cup (E14-01R), $30 \mathrm{~mm}$ long by $40 \mathrm{~mm}$ wide, was centered between the left-side and right-side walls $20 \mathrm{~mm}$ from the floor. Two retractable levers (E23-17RA), requiring a force of $0.2 \mathrm{~N}$ to operate, were mounted on the front wall of each chamber $70 \mathrm{~mm}$ above the floor; the levers were $30 \mathrm{~mm}$ wide, and the edge of each lever was $25 \mathrm{~mm}$ from its respective left and right sidewalls. Two $24-\mathrm{V}$ DC stimulus lights (H11-03R) were installed $35 \mathrm{~mm}$ above the levers. A food dispenser (H14-23R) located behind the front wall delivered 45-mg grain pellets (BioServ) into the food cup. A third, nonretractable lever (H21-03R), requiring a force of $0.2 \mathrm{~N}$ to operate, was centered on the rear wall of each chamber, $60 \mathrm{~mm}$ above the floor. A 24-V DC houselight (H11-01R) centered on the rear wall $20 \mathrm{~mm}$ below the ceiling provided the illumination of the chamber. A $26 \times 40 \mathrm{~mm}$ speaker (H1201R) mounted on the rear wall, $10 \mathrm{~mm}$ from the left sidewall and $65 \mathrm{~mm}$ from the house light, was connected to a white noise generator (E12-08) providing a constant white noise at $20 \mathrm{kHz}( \pm 3 \mathrm{~dB})$. All experimental events were programmed and data recorded in a separate room by two Windowscontrolled computers using Coulbourn Instruments software (Graphic State Notation, version 3.03) and interfacing equipment operating at a 0.01 -s resolution.

\section{Procedure}

Training Four 45-mg pellets were placed into the food cup, and training began with the left lever being extended into the chamber. A fixed-ratio schedule of one response (FR 1) was associated with the extended lever, but there were no attempts to shape manually the response of pressing on it. The FR 1 was operative until the rats had pressed on the extended lever to produce 60 food pellets, or until $30 \mathrm{~min}$ had elapsed, whichever happened first. When the rats pressed consistently on that lever to produce 60 food pellets in two consecutive sessions, it was retracted from the chamber and the right lever was extended into the chamber; responding on the right lever was trained in a similar fashion over the following days. Finally, the rear lever was reinstalled in the chamber with an FR 1 schedule associated with it, and the other two levers were retracted from the chamber. Once the rats had pressed on the rear lever to produce 60 food pellets in two consecutive sessions, the front levers were extended into the chamber, and training ended in a 30-min session with the rear and the two front levers all providing food pellets according to a concurrent FR 1 FR 1 FR 1 schedule of reinforcement.

General procedure A concurrent-chains procedure similar to that described in detail elsewhere (Aparicio et al., 2013) was used, the only difference being that the choice link used two random-interval (RI) schedules instead of one RI schedule, and the terminal links used fixed-time (FT) responseindependent schedules to deliver the SSR and the LLR. Each session consisted of a series of 60 choice cycles, organized in six delay components of ten cycles each that initiated with the houselight turning on. A single response on the rear lever (a) turned off the houselight, (b) extended the front levers into the chamber, and (c) turned on the stimulus lights above them, advancing the procedure to the choice link; two nonindependent RI 12-s schedules, one associated with the left lever and the other with the right lever, arranged equal numbers of left and right terminal-link entries (Alsop \& Davison, 1986; Stubbs \& Pliskoff, 1969). Once an entry was set up for the left or the right lever, the first response on that lever advanced the procedure to its terminal link and retracted the other, inoperative lever from the chamber, turning off the light above it. The second press on the available lever started the FT; that lever was not retracted from the chamber, in order to avoid signaling the delay to the LLR (i.e., its function as a conditioned reinforcement). Further presses on the same lever during the delay or at the end of it had no programmed consequences. For half of the rats within each strain, the terminal link delivering one pellet (the SSR) was associated with the left lever, and the terminal link delivering four pellets (the LLR) with the right lever; these relations were reversed for the other half of the rats within each strain (i.e., the LLR was associated with the left lever and the SSR with the right lever). The terminal link delivering the SSR remained constant at a FT $0.1 \mathrm{~s}$ (i.e., the minimal unit of time for the program to move from one state to another), and the terminal link delivering the LLR was a FT that every ten cycles took on a different value $(0.1,5,10,20,40$, or $80 \mathrm{~s})$. Upon delivery of either the SSR or the LLR, each cycle ended by (a) retracting the operative 
lever, (b) turning off the stimulus light above it, and (c) turning on the houselight to start a new cycle. Each delay component of ten cycles was followed by a 60 -s blackout during which responses on the rear lever were not effective, all lights were extinguished, and the front levers were retracted from the chamber. Sessions were programmed to end after 60 cycles (i.e., six delay components) or $60 \mathrm{~min}$, whichever occurred first. Most sessions, however, ended after 60 cycles, not requiring the 60 -min maximum time to end the session. Delay components were presented within sessions in ascending, descending, or random order, with each presentation order of delay conditions lasting for 105 sessions. All rats were exposed to the same sequence of ascending, descending, and random conditions. Then, a replication of the ascending presentation order of delay conditions (reascending) was scheduled for another 105 sessions. Sessions were conducted seven days per week at about the same time each day.

\section{Data analysis}

For each data set of 105 sessions, corresponding to the ascending, descending, random, and redetermination-of-ascendingorder conditions, data were analyzed across blocks of sessions and also across reinforcers within a component. All computations used choice-link responses emitted on the LL lever and the SS lever. The analysis across blocks of sessions organized each data set into seven blocks of 15 days each, which in our experience is a large enough number of days to observe consistent changes in choice. Responses on the LL and SS levers were counted separately for each delay and aggregated across sessions of the same block. Then, the corresponding proportions of LL choice [LL responses / (LL responses + SS responses)] and ratios of responses (LL/SS) were calculated using computations for individuals within each strain and the group's mean. For the analysis across reinforcers within a component, we pooled the data across all 105 sessions of the same condition, counting responses on the LL and SS levers separately for each reinforcer within the same delay component, regardless of whether it was a SSR or LLR; accordingly, for each delay component ten proportions of LL choices and ten ratios of responses could be computed, or 60 proportions of LL choices and 60 response ratios for the six delay components. These computations were obtained for each rat within a strain and used to calculate the means and standard deviations of the groups.

The distribution of responses across the two levers in the initial or choice link was analyzed with the general form of the matching law (Baum, 1974; Baum \& Rachlin, 1969):

$\log \left(\frac{B_{1}}{B_{2}}\right)=s^{*} \log \left(\frac{r_{1}}{r_{2}}\right)+\log b$,

where $B_{1}$ and $B_{2}$ are the behavior allocations, measured in time or responses (i.e., LL responses and SS responses, respectively), to Alternatives 1 and $2 ; r_{1}$ and $r_{2}$ are the rates of reinforcement, amounts of reinforcement, or immediacies of reinforcement (reciprocal of delay of reinforcement) produced by responding at Alternatives 1 and $2 ; b$ is a measure of bias toward one alternative or the other arising from factors other than $r_{1}$ and $r_{2}$; and $s$ is the sensitivity of the behavior ratio to the ratio of the values (i.e., rates, amounts, or immediacies of reinforcement) of the activities (Baum \& Rachlin, 1969). The ratios of responses (LL/SS) and delays to food delivery (SS/ LL) were transformed into base-2 logarithms. Linear regression analysis (the least squares method) was conducted with the $\log _{2}$ of the response ratio plotted against the $\log _{2}$ of the delay ratio $(\mathrm{SS} / \mathrm{LL})$. The resulting slope was used to estimate the sensitivity of choices to changes in the immediacy of the reinforcement (i.e., the reciprocal of the delay to reinforcement), and the $y$-intercept to estimate bias (Baum, 1974; Baum \& Rachlin, 1969).

Equation 1 was entered into the Origin software (version 8.5) as a user-defined equation, providing nonlinear curve fitting to the proportions of LL choices; $A$ was free to vary (i.e., it was not assumed to be $1.0 \mathrm{LL}$ choice at the $y$-intercept), and the value of the parameter $k$ was used to estimate the rate of discounting.

The area under the empirical discounting curve (AUC) was computed using Myerson, Green, and Warusawitharana's (2001) method, providing a theory-free estimate of delay discounting; it was expressed as a proportion of 1.0 , with values close to 1.0 indicating minimal or no discounting, and values close to zero maximal discounting. Nonparametric statistical Mann-Whitney $U$ tests and Wilcoxon signed ranks tests were used to examine the differences between strains in estimates of $k$ (Eq. 1), bias, slopes from regression lines (Eq. 2), and AUC computed for the data of the individual LEWs and F344s. Linear curve fitting and nonparametric statistical tests, at the alpha level of .05, were implemented using Origin.

\section{Results}

\section{Hyperbolic-decay functions}

The group means of proportions of LL choices are plotted in Figs. 1 and 2 as a function of delay to the LLR. From top to bottom, the graphs show the data for each block of sessions (Fig. 1) and each food delivery (Fig. 2); from left to right, the figures show the data obtained in the ascending, descending, random, and reascending conditions. Continuous and dashed lines are the best fits using Eq. 1 to the data points of the LEWs (circles) and F344s (squares), respectively. Generally, the groups' means of the proportions of LL choices decreased with increasing delays to LLR. All delay conditions show that 

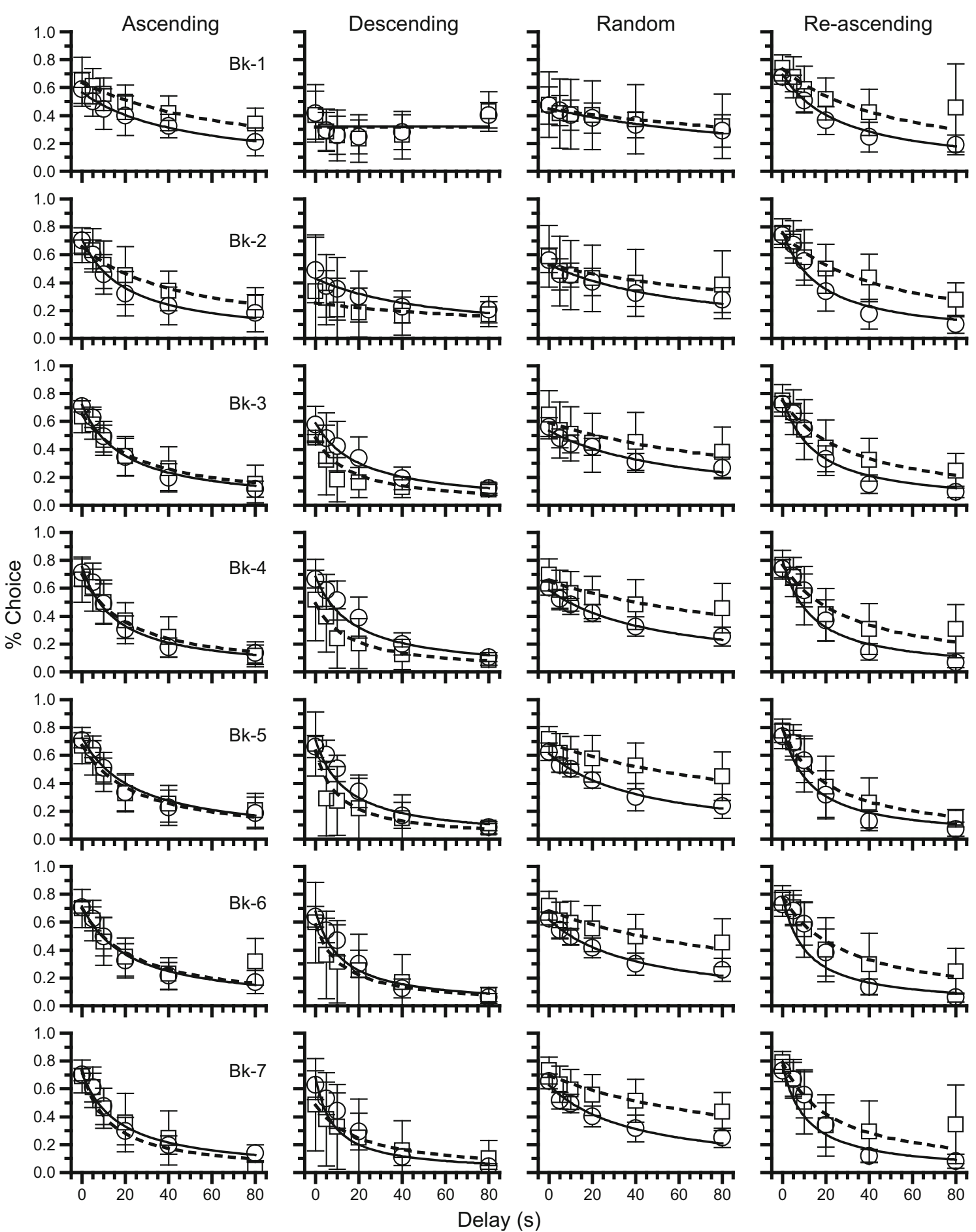

Fig. 1 Proportions of LL choices [LL / $(L L+S S)]$ as a function of delay (in seconds) to the LLR. Panels show the group means among the Lewis (LEW, circles) and Fischer 344 (F344, squares) rats for the ascending,

delay discounting increased across blocks of sessions and food deliveries, with a tendency of the LEWs to choose the SS lever more that the F344s, but this difference was unreliable.

The resulting parameters for the $y$-intercept $(A), k$, and $R^{2}$, computed for blocks of sessions, are presented in Table 1 , descending, random, and reascending delay-order conditions. Lines that extend from the squares and circles correspond to standard deviations. Bk stands for blocks of sessions

and those same parameters computed for food deliveries are in Table 2. The delay-discounting functions of the ascending

Fig. 2 Proportions of LL choices [LL / (LL + SS)] as a function of delay (in seconds) to the LLR. F stands for food delivery. Other details are as in Fig. 1 

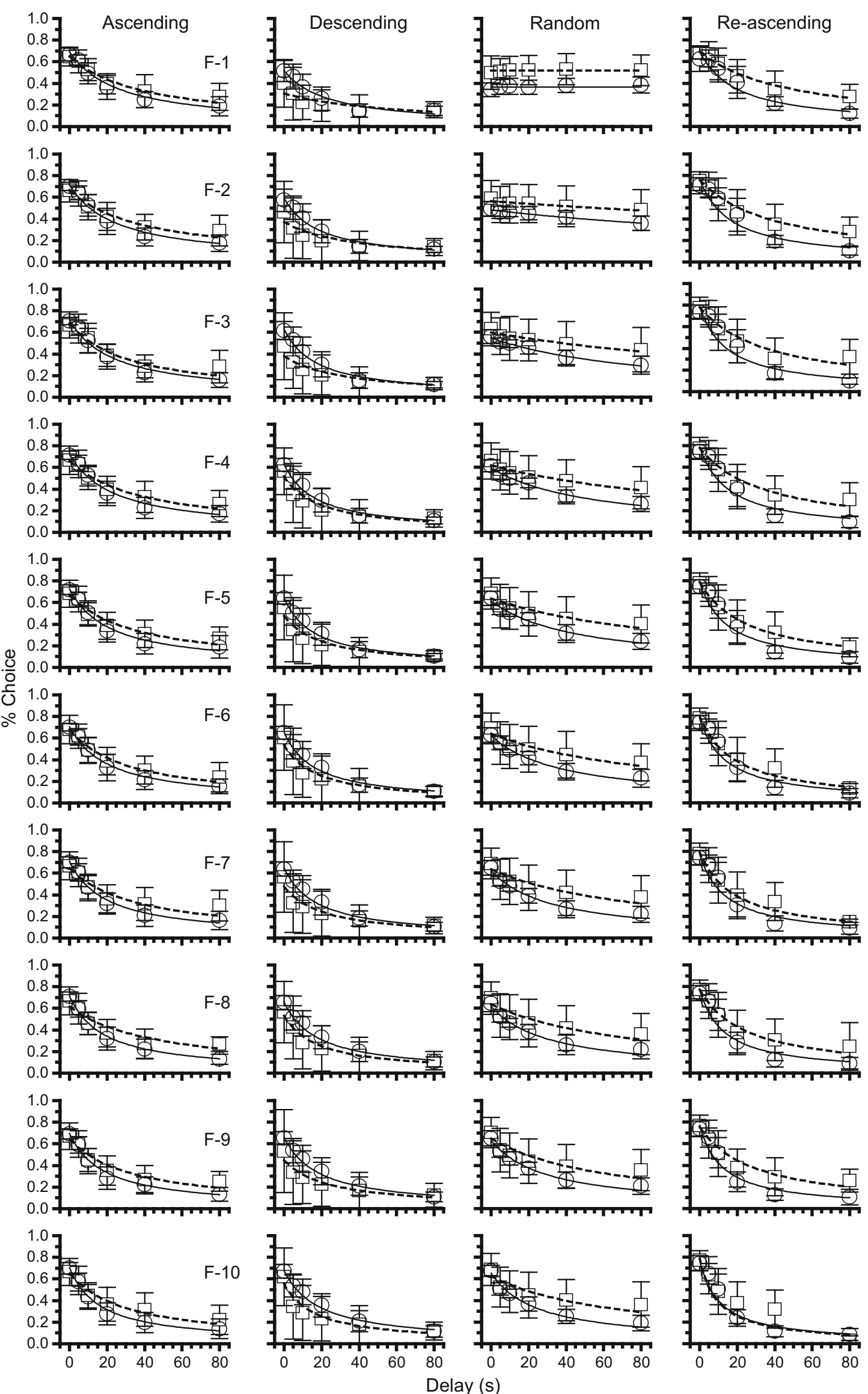
Table 1 Hyperbolic discounting, parameters (Eq. 1) computed for blocks of sessions

\begin{tabular}{|c|c|c|c|c|c|c|c|c|}
\hline \multirow[t]{2}{*}{ Block } & \multicolumn{2}{|c|}{ Ascending } & \multicolumn{2}{|c|}{ Descending } & \multicolumn{2}{|c|}{ Random } & \multicolumn{2}{|c|}{ Reascending } \\
\hline & LEW & F344 & LEW & F344 & LEW & F344 & LEW & F34 \\
\hline \\
\hline 1 & .567 & .635 & .320 & .317 & .451 & .447 & .696 & .733 \\
\hline 2 & .714 & .659 & .430 & .253 & .531 & .527 & .767 & .753 \\
\hline 3 & .724 & .655 & .592 & .485 & .534 & .591 & .766 & .750 \\
\hline 4 & .757 & .704 & .687 & .494 & .589 & .649 & .805 & .768 \\
\hline 5 & .729 & .676 & .713 & .637 & .613 & .6 & .802 & .794 \\
\hline 6 & .716 & .701 & .680 & .586 & .619 & .6 & .788 & .783 \\
\hline 7 & .712 & .736 & .686 & .486 & .628 & .696 & .772 & .794 \\
\hline Mean & .703 & .681 & .587 & .465 & .567 & .610 & .771 & .768 \\
\hline \\
\hline 1 & .020 & .013 & .000 & .000 & .008 & .005 & .036 & .017 \\
\hline 2 & .049 & .021 & .017 & .008 & .014 & .007 & .058 & .021 \\
\hline 3 & .053 & .039 & .048 & .062 & .016 & .009 & .065 & .031 \\
\hline 4 & .065 & .049 & .058 & .066 & .020 & .008 & .076 & .032 \\
\hline 5 & .041 & .041 & .071 & .098 & .023 & .008 & .080 & .049 \\
\hline 6 & .047 & .042 & .084 & .083 & .024 & .008 & .093 & .034 \\
\hline 7 & .058 & .082 & .121 & .049 & .025 & .009 & .091 & .044 \\
\hline Mean & .048 & .041 & .057 & .052 & .018 & .008 & .071 & .033 \\
\hline \multicolumn{9}{|l|}{$R^{2}$} \\
\hline 1 & .983 & .977 & -.250 & -.250 & .942 & .845 & .974 & .904 \\
\hline 2 & .982 & .986 & .853 & .373 & .929 & .594 & .967 & .987 \\
\hline 3 & .985 & .986 & .992 & .979 & .935 & .748 & .959 & .982 \\
\hline 4 & .963 & .976 & .982 & .966 & .982 & .709 & .934 & .940 \\
\hline 5 & .961 & .956 & .959 & .933 & .981 & .832 & .992 & .991 \\
\hline 6 & .986 & .855 & .954 & .981 & .978 & .808 & .921 & .970 \\
\hline 7 & .991 & .916 & .918 & .998 & .945 & .865 & .935 & .924 \\
\hline Mean & .978 & .950 & .773 & .712 & .956 & .772 & .955 & .957 \\
\hline
\end{tabular}

condition show that Eq. 1 provided excellent fits to the data of the LEWs and F344s across blocks of sessions, means $R^{2} \mathrm{~S}=.978$ and .950 , respectively, and food deliveries, mean $R^{2} \mathrm{~S}=.985$ and .912 , respectively; $y$-intercepts $(A)$ for the data of the LEWs across blocks and food deliveries (means of .703 and .711, respectively) were higher than those for the data of the F344s (means of .587 and .657, respectively).

As is shown in Table 1, Blocks 1-2 of the ascending condition show steeper discounting functions for the data of the LEWs $(k=.020$ and .049$)$ than for the F344s $(k=.013$ and .021 ), indicating that the LEWs chose more impulsively than the F344s. Both strains, however, produced similar discounting functions in Blocks 3-7. Estimates of $k$, ranging from .020 to $.065(M=.048)$ for the data of the LEWs, were not significantly different $(W=22, p>.05)$ from those for the data of the F344s, ranging from .013 to $.082(M=.041)$. Figure 2 shows that the discounting functions generated by the LEWs for each food
Table 2 Hyperbolic discounting, parameters (Eq. 1) computed for food deliveries

\begin{tabular}{|c|c|c|c|c|c|c|c|c|}
\hline \multirow[t]{2}{*}{ Food } & \multicolumn{2}{|c|}{ Ascending } & \multicolumn{2}{|c|}{ Descending } & \multicolumn{2}{|c|}{ Random } & \multicolumn{2}{|c|}{ Reascending } \\
\hline & LEW & F344 & LEW & F344 & LEW & F344 & LEW & F344 \\
\hline \multicolumn{9}{|l|}{$A$} \\
\hline 1 & .695 & .648 & .521 & .305 & .365 & .516 & .696 & .691 \\
\hline 2 & .714 & .665 & .594 & .371 & .482 & .562 & .786 & .760 \\
\hline 3 & .713 & .685 & .631 & .380 & .547 & .598 & .793 & .782 \\
\hline 4 & .724 & .665 & .634 & .519 & .594 & .620 & .793 & .779 \\
\hline 5 & .729 & .671 & .647 & .478 & .615 & .638 & .793 & .790 \\
\hline 6 & .707 & .681 & .659 & .535 & .624 & .640 & .786 & .806 \\
\hline 7 & .709 & .647 & .652 & .476 & .623 & .638 & .779 & .794 \\
\hline 8 & .717 & .614 & .660 & .534 & .619 & .638 & .777 & .776 \\
\hline 9 & .700 & .646 & .664 & .448 & .619 & .651 & .778 & .757 \\
\hline 10 & .707 & .651 & .679 & .557 & .656 & .624 & .780 & .798 \\
\hline Mean & .711 & .657 & .634 & .460 & .575 & .612 & .776 & .773 \\
\hline \multicolumn{9}{|l|}{$k$} \\
\hline 1 & .038 & .025 & .044 & .016 & .000 & .000 & .050 & .020 \\
\hline 2 & .040 & .024 & .054 & .028 & .004 & .002 & .060 & .024 \\
\hline 3 & .042 & .031 & .058 & .030 & .011 & .005 & .068 & .027 \\
\hline 4 & .043 & .026 & .062 & .055 & .018 & .008 & .065 & .028 \\
\hline 5 & .047 & .028 & .064 & .050 & .022 & .010 & .070 & .039 \\
\hline 6 & .048 & .032 & .063 & .058 & .026 & .011 & .072 & .055 \\
\hline 7 & .051 & .027 & .059 & .048 & .030 & .013 & .073 & .053 \\
\hline 8 & .055 & .021 & .057 & .057 & .032 & .013 & .079 & .040 \\
\hline 9 & .055 & .030 & .055 & .042 & .032 & .017 & .084 & .034 \\
\hline 10 & .062 & .032 & .053 & .060 & .041 & .014 & .096 & .106 \\
\hline Mean & .048 & .028 & .057 & .044 & .022 & .009 & .072 & .043 \\
\hline \multicolumn{9}{|l|}{$R^{2}$} \\
\hline 1 & .972 & .942 & .927 & .601 & -.250 & -.251 & .953 & .987 \\
\hline 2 & .980 & .935 & .974 & .747 & .958 & .688 & .942 & .970 \\
\hline 3 & .984 & .919 & .987 & .856 & .980 & .835 & .946 & .939 \\
\hline 4 & .986 & .930 & .993 & .893 & .967 & .858 & .941 & .953 \\
\hline 5 & .980 & .916 & .997 & .918 & .975 & .817 & .938 & .993 \\
\hline 6 & .990 & .959 & .994 & .936 & .989 & .820 & .949 & .987 \\
\hline 7 & .985 & .831 & .992 & .904 & .973 & .770 & .951 & .991 \\
\hline 8 & .997 & .857 & .993 & .953 & .974 & .770 & .952 & .978 \\
\hline 9 & .989 & .890 & .995 & .908 & .974 & .770 & .942 & .955 \\
\hline 10 & .990 & .943 & .994 & .905 & .974 & .714 & .950 & .928 \\
\hline Mean & .985 & .912 & .985 & .862 & .851 & .679 & .946 & .968 \\
\hline
\end{tabular}

delivery of the ascending condition were similar to those of the F344s (i.e., the continuous and dotted lines are close to one another). Yet, the proportions of LL choices generated by the F344s with delays to LLR higher than $20 \mathrm{~s}$ were slightly higher than those produced by the LEWs with the same delays, indicating that food-by-food, the F344s chose less impulsively across delays to LLR. Estimates of $k$ for the group data of the LEWs, ranging from .038 to $.062(M=.048)$ were significantly 
higher $(W=55, p<.05)$ than the estimates for the $\mathrm{F} 344 \mathrm{~s}$, ranging from .021 to $.032(M=.028)$.

As is shown in the top graph of Fig. 1 for the descending condition, the pattern of choices that both strains established in ascending order was disrupted by delays occurring within sessions in descending order. For both strains, Block 1 of the descending condition shows flat slopes $(k=.0)$ and aberrant fits $\left(R^{2}=-.250\right)$. Block 2 shows a steeper discounting function for the LEWs $(k=.017)$ than for the F344s $(.008)$; the former strain recovered the pattern of choice shown in the ascending condition faster $\left(R^{2}=.853\right)$ than the latter $\left(R^{2}=.373\right)$. In Blocks $3-5$, the discounting functions of the F344s are steeper ( $k=.062, .066$, and .098 , respectively) than those of the LEWs ( $k=.048, .058$, and .071 , respectively), indicating that the former chose more impulsively than the latter strain. Differences in the discounting functions between both strains vanished in Block 6 ( $k=.080$ vs. .083 , respectively). Still, in Block 7 the LEWs generated the steepest discounting function $(k=.121)$. Estimates of $k$ ranging from .000 to $.121(M=.057)$ for the data of the LEWs were not significantly different $(W=$ $13, p>.05$ ) from those for the F344s, ranging from .000 to $.098(M=.052)$, as is shown in Table 1. Even though Fig. 2 shows similar discounting functions for each food delivery of the descending condition (i.e., the continuous and dotted lines are close to one another), the discounting functions of the LEWs were steeper than those of the F344s. Following Table 2, estimates of $k$ for the data of the LEWs, ranging from .044 to $.064(M=.057)$ were significantly higher $(W=50, p<$ $.05)$ than those for the F344s, ranging from .016 to .060 ( $M=$ .044 ), showing that with delays being presented within sessions in descending order, food by food, the LEWs chose more impulsively than the F344s.

As is shown in Tables 1 and 2, with delay components presented within sessions in random order, Eq. 1 also provided good fits to the group data of both strains across blocks of sessions (mean $R^{2} \mathrm{~S}=.956$ and .772 , respectively) and food deliveries (mean $R^{2} \mathrm{~s}=.851$ and .679 , respectively). The $y$ intercept $(A)$ was higher for the data of the F344s than for the LEWs across blocks of sessions (mean $A \mathrm{~s}=.610$ and .567 , respectively) and food deliveries (mean $A \mathrm{~s}=.612$ and .575 , respectively).

Block 1 shows that both strains generated similar discounting functions ( $k=.008$ and .005 , respectively) responding to delays to the LLR presented in a random order (see Table 1). Blocks 2-7, however, show steeper discounting functions for the group data of the LEWs; estimates of $k$ for the LEWs, ranging from .008 to $.025(M=.018)$ were significantly higher $(W=28, p<.05)$ than those for the $\mathrm{F} 344 \mathrm{~s}$, ranging from .005 to $.009(M=.008)$. For the first food delivery of the random condition, Fig. 2 shows flat discounting functions $(k=0)$, resulting in aberrant fits $\left(R^{2}=-.251\right)$ to data points of the group data of both strains. Consistent with the results for blocks of sessions, Food Deliveries 2-10 show steeper discounting functions for the group data of the LEWs (see Table 2); estimates of $k$ for the data of the LEWs, ranging from .000 to $.041(M=.022)$, were significantly higher $(W=45, p<.05)$ than those corresponding to the $\mathrm{F} 344 \mathrm{~s}$, ranging from .000 to $.017(M=.009)$.

The results of the reascending condition were close to those obtained during the first ascending determination. As is shown in Tables 1 and 2, Eq. 1 provided excellent fits to the group data of both strains across blocks of sessions (mean $R^{2} \mathrm{~s}=.955$ and .957 , respectively) and food deliveries (mean $R^{2} \mathrm{~s}=.946$ and .968 , respectively); the $y$-intercepts for the data of the LEWs across blocks of sessions (mean $A=.771$ ) and food deliveries (mean $A=.776$ ) were similar to those corresponding to the data of the F344s (mean $A \mathrm{~s}=.768$ and .773 , respectively). The reascending condition shows steeper discounting functions for both strains, but the LEWs made more impulsive choices than the F344s. Estimates of $k$ for the data of the LEWs, ranging from .036 to $.093(M=.071)$, were significantly higher $(W=28, p>.05)$ than those for the F344s, ranging from .017 to $.049(M=.033)$. The food-by-food analysis revealed similar results, with Food Deliveries 1-9 showing steeper discounting functions for the LEWs than for the F344s (see Fig. 2). Nevertheless, the discounting function (see Table 2) that the F344s showed in Food Delivery 10 was steeper $(k=.106)$ than that of the LEWs $(k=.096)$; estimates of $k$ for the LEWs, ranging from .050 to $.096(M=.072)$, were significantly higher $(W=54, p<.05)$ than those for the F344s, ranging from .020 to $.106(M=.043)$.

In summary, the LEWs in the ascending condition responded more impulsively than did F344s for the first two blocks, and with further training this difference in impulsive choices disappeared. From all of the orders of delay to LLR presentation, the descending order generated the most disruption of response patterns, as evidenced by the lowest sensitivity and goodness of fit across delay presentation orders; the LEWs adapted faster to the descending condition. In the random condition, strain differences were not as evident in the first two blocks, but with training the LEWs made more impulsive choices than did the F344s. The same trend was observed during the reascending condition, replicating what had been found in the ascending condition; such differences between strains were maintained across blocks and food deliveries.

\section{General form of the matching law}

The $\log _{2}$ of response ratios (LL/SS) is plotted in Figs. 3 and 4 against the $\log _{2}$ of delay ratios (SS/LL). From top to bottom, the graphs show the data for each block of sessions (Fig. 3) and each food delivery (Fig. 4); from left to right, the data obtained in the ascending, descending, random, and reascending presentation-order-of-delays conditions. The dotted lines intercepting the $y$-axis at zero are the indifference 

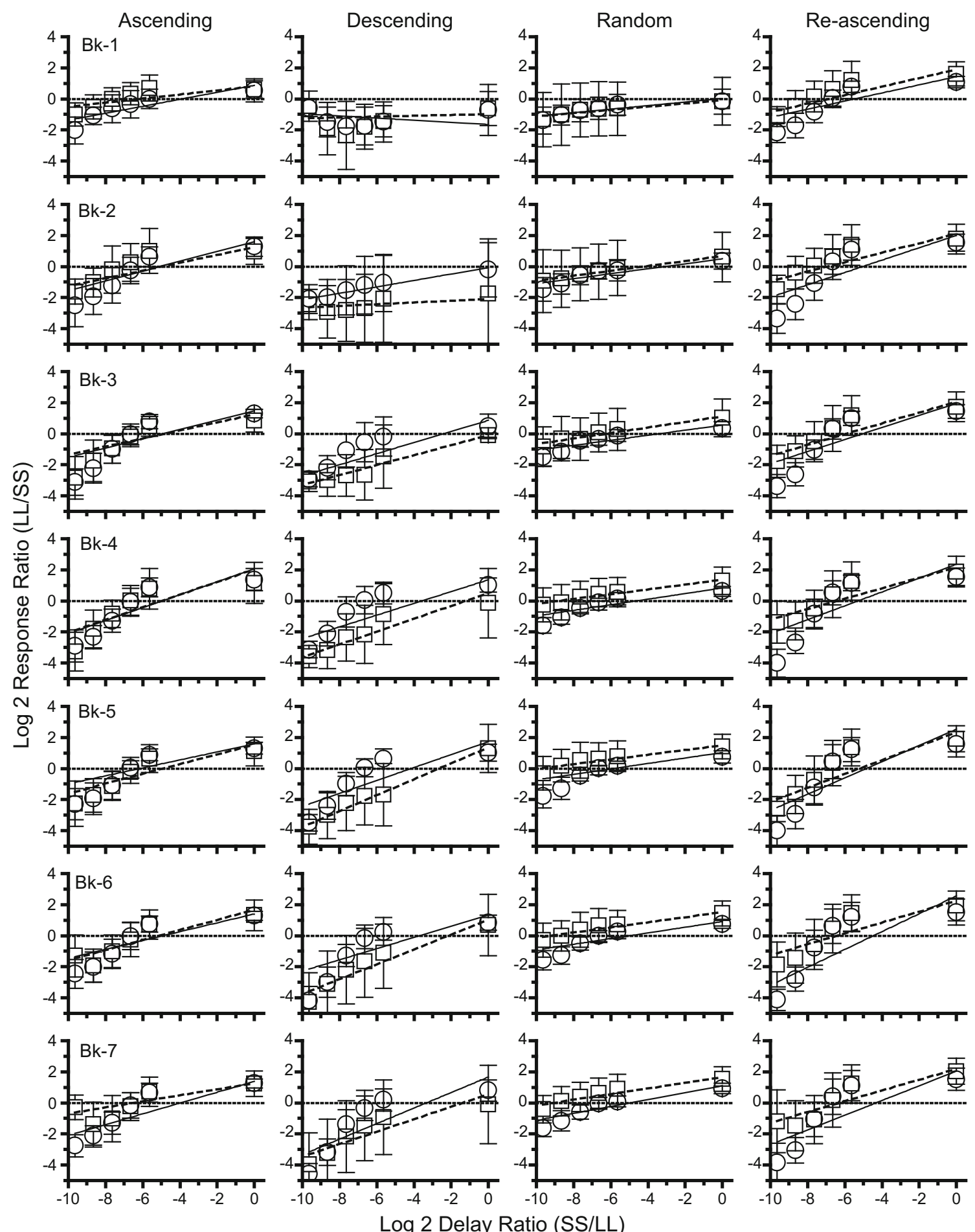

Fig. $3 \log _{2}$ of response ratios (LL/SS) as a function of the $\log _{2}$ of delay ratios (SS/LL), presented for each block of sessions. Other details are as in Fig. 1

lines. The continuous and dashed lines are the best fits using Eq. 2 to the group data points of the LEW (circles) and F344 (squares) rats, respectively. The resulting parameters for bias (b), slope (s), and $R^{2}$ computed for each block of sessions are presented in Table 3, and the same parameters calculated for each food delivery are in Table 4.
Figure 3 shows a positive relation between the log of response ratios and the log of delay ratios, indicating sensitivity of preference to within-session changes in the ratio of immediacy to reinforcement (i.e., the reciprocal of the delay to reinforcement). Equation 2 provided good fits to the response ratios that both strains of rats produced with delays to LLR 



Fig. $4 \log _{2}$ of response ratios (LL/SS) as a function of the $\log _{2}$ of delay ratios (SS/LL). F stands for food delivery. Other details are as in Fig. 1 
Table 3 General form of the matching law (Eq. 2), parameters for each block of sessions

\begin{tabular}{|c|c|c|c|c|c|c|c|c|}
\hline \multirow[t]{2}{*}{ Block } & \multicolumn{2}{|c|}{ Ascending } & \multicolumn{2}{|c|}{ Descending } & \multicolumn{2}{|c|}{ Random } & \multicolumn{2}{|c|}{ Reascending } \\
\hline & LEW & F344 & EW & F344 & EW & F344 & LEW & F34 \\
\hline \multicolumn{9}{|l|}{ Bias } \\
\hline 1 & 0.873 & 0.808 & -1.650 & -0.981 & 0.026 & -0.080 & 1.428 & 1.89 \\
\hline 2 & 1.593 & 1.249 & -0.073 & -2.097 & 0.491 & 0.673 & 2.000 & 2.112 \\
\hline 3 & 1.471 & 1.247 & 0.858 & -0.099 & 0.552 & 1.116 & 1.953 & 2.059 \\
\hline 4 & 2.059 & 2.033 & 1.355 & 0.510 & 0.832 & 1.360 & 2.358 & 2.163 \\
\hline 5 & 1.583 & 1.543 & 1.732 & 1.365 & 1.029 & 1.498 & 2.531 & 2.341 \\
\hline 6 & 1.400 & 1.662 & 1.332 & 1.036 & 0.910 & 1.516 & 2.563 & 2.25 \\
\hline 7 & 1.381 & 1.257 & 1.668 & 5 & 1.098 & 1.660 & 2.047 & 2.18 \\
\hline To & 1.480 & 1.400 & 16 & 41 & 0.705 & 1.106 & 2.126 & 2.144 \\
\hline \\
\hline 1 & .225 & .131 & -.069 & 27 & .114 & .105 & .261 & .274 \\
\hline 2 & .3 & & & & .159 & & .397 & .305 \\
\hline 3 & .299 & .259 & .357 & .320 & .163 & & .387 & .347 \\
\hline 4 & .415 & .406 & .379 & & .180 & .155 & .445 & .336 \\
\hline 5 & .257 & .315 & .417 & 3 & .176 & .152 & .522 & .447 \\
\hline 6 & .285 & .323 & .361 & .478 & .183 & .164 & .574 & .35 \\
\hline 7 & .348 & .194 & .500 & .401 & .222 & .182 & .473 & .346 \\
\hline Mear & .306 & .268 & .307 & .315 & .171 & .156 & .437 & .34 \\
\hline \multicolumn{9}{|l|}{$R^{2}$} \\
\hline 1 & .729 & .523 & -.129 & -.221 & .694 & .831 & .563 & .80 \\
\hline 2 & .687 & .721 & .943 & -.042 & .790 & .972 & .605 & .76 \\
\hline 3 & .634 & .524 & .818 & .994 & .720 & .923 & .568 & .81 \\
\hline 4 & .637 & .588 & .736 & .872 & .697 & .959 & .773 & .79 \\
\hline 5 & .527 & .683 & .599 & .985 & .558 & .886 & .579 & .79 \\
\hline 6 & .713 & .717 & .534 & .899 & .708 & .918 & .632 & .72 \\
\hline 7 & .824 & .618 & .613 & .753 & .824 & .895 & .634 & .81 \\
\hline Mean & .679 & .625 & .588 & .606 & .713 & .912 & .622 & .78 \\
\hline
\end{tabular}

being presented in ascending order, accounting for changes in preference that occurred as a function of rapid changes in the delay ratio (mean $R^{2} \mathrm{~s}=.679$ and .625 , respectively). Estimates of bias for the data of the LEWs, ranging from 0.873 to $2.059(M=1.480)$, were not significantly different $(W=22, p>.05)$ from those for the $\mathrm{F} 344 \mathrm{~s}$, ranging from 0.808 to $2.033(M=1.400)$. In Blocks 1 and 2, the data points of the F344s are close to or above the indifference line, indicating indifference or a slight preference for the LL; in contrast, most data points of the LEWs fall below the indifference line, showing preference for the SS lever. Blocks 3-7 show that both strains established similar patterns of choice responding to delays to LLR presented in ascending order. Indifference occurred when the LLR was delayed $10 \mathrm{~s}$ (delay ratio -6.64), preference for the SS lever when it was delayed $20 \mathrm{~s}$ or longer (delay ratios $-7.64,-8.64$, and -9.64 ), and preference for the LL lever when the LLR was delayed 5 or 0.1 s (i.e., delay ratios -5.64 and 0 ). Estimates of sensitivity to
Table 4 General form of the matching law (Eq. 2), parameters for each food delivery

\begin{tabular}{|c|c|c|c|c|c|c|c|c|}
\hline \multirow[t]{2}{*}{ Food } & \multicolumn{2}{|c|}{ Ascending } & \multicolumn{2}{|c|}{ Descending } & \multicolumn{2}{|c|}{ Random } & \multicolumn{2}{|c|}{ Reascending } \\
\hline & LEW & F344 & LEW & F344 & LEW & F344 & LEW & F344 \\
\hline \multicolumn{9}{|l|}{ Bias } \\
\hline 1 & 1.455 & 1.166 & 0.424 & -0.688 & -0.973 & 0.001 & 1.635 & 1.254 \\
\hline 2 & 1.417 & 1.312 & 1.054 & 0.118 & 0.076 & 0.614 & 2.013 & 1.911 \\
\hline 3 & 1.366 & 1.526 & 1.097 & 0.162 & 0.609 & 0.931 & 2.387 & 2.248 \\
\hline 4 & 1.474 & 1.548 & 0.970 & 0.554 & 0.937 & 1.259 & 2.383 & 2.300 \\
\hline 5 & 1.532 & 1.657 & 1.049 & 0.648 & 1.148 & 1.343 & 2.417 & 2.472 \\
\hline 6 & 1.321 & 1.742 & 1.118 & 1.365 & 1.072 & 1.375 & 2.319 & 2.754 \\
\hline 7 & 1.397 & 1.401 & 1.126 & 0.636 & 1.248 & 1.315 & 2.230 & 2.615 \\
\hline 8 & 1.462 & 1.502 & 1.150 & 0.886 & 1.274 & 1.386 & 2.270 & 2.125 \\
\hline 9 & 1.333 & 1.530 & 1.141 & 0.570 & 1.284 & 1.454 & 2.253 & 2.097 \\
\hline 10 & 1.390 & 1.408 & 1.297 & 1.098 & 1.304 & 1.321 & 2.307 & 2.042 \\
\hline Mean & 1.415 & 1.479 & 1.043 & 0.535 & 0.798 & 1.100 & 2.221 & 2.182 \\
\hline \multicolumn{9}{|l|}{$s$} \\
\hline 1 & .249 & .212 & .285 & .214 & -.026 & -.018 & .376 & .205 \\
\hline 2 & .253 & .229 & .381 & .323 & .063 & .059 & .424 & .290 \\
\hline 3 & .264 & .275 & .379 & .329 & .119 & .109 & .489 & .326 \\
\hline 4 & .275 & .270 & .368 & .374 & .175 & .162 & .473 & .339 \\
\hline 5 & .277 & .294 & .383 & .402 & .195 & .186 & .482 & .425 \\
\hline 6 & .283 & .307 & .356 & .480 & .200 & .203 & .494 & .515 \\
\hline 7 & .295 & .261 & .348 & .391 & .239 & .205 & .473 & .517 \\
\hline 8 & .328 & .297 & .332 & .417 & .244 & .221 & .492 & .352 \\
\hline 9 & .314 & .304 & .338 & .382 & .262 & .242 & .488 & .366 \\
\hline 10 & .323 & .283 & .326 & .440 & .267 & .223 & .515 & .344 \\
\hline Mean & .286 & .273 & .350 & .375 & .174 & .159 & .471 & .368 \\
\hline \multicolumn{9}{|l|}{$R^{2}$} \\
\hline 1 & .474 & .649 & .716 & .881 & .834 & .642 & .551 & .660 \\
\hline 2 & .593 & .650 & .758 & .948 & .480 & .756 & .591 & .730 \\
\hline 3 & .651 & .628 & .791 & .935 & .472 & .854 & .629 & .750 \\
\hline 4 & .683 & .665 & .826 & .962 & .633 & .859 & .601 & .768 \\
\hline 5 & .675 & .726 & .848 & .982 & .611 & .922 & .593 & .779 \\
\hline 6 & .725 & .692 & .793 & .980 & .602 & .935 & .642 & .787 \\
\hline 7 & .712 & .739 & .741 & .968 & .692 & .953 & .634 & .846 \\
\hline 8 & .760 & .817 & .745 & .904 & .688 & .950 & .657 & .822 \\
\hline 9 & .750 & .764 & .775 & .956 & .737 & .972 & .657 & .895 \\
\hline 10 & .761 & .747 & .726 & .996 & .789 & .979 & .674 & .876 \\
\hline Mean & .678 & .708 & .772 & .951 & .654 & .882 & .623 & .791 \\
\hline
\end{tabular}

the immediacy of reinforcement $(s)$ for the data of the LEWs, ranging from .225 to $.415(M=.306)$, were not significantly different $(W=22, p>.05$ ) from those for the $\mathrm{F} 344 \mathrm{~s}$, ranging from .131 to $.406(M=.268)$.

With delays to LLR presented in descending order (see Table 3), estimates of bias ranging from -1.650 to 1.732 ( $M=$ 0.746 ) for the data of the LEWs were not significantly different $(W=25, p>.05)$ from those for the $\mathrm{F} 344 \mathrm{~s}$, ranging from 2.097 to $1.365(M=0.041)$. Equation 2 provided good fits to 
group data points of both strains, accounting for changes in preference as a function of changes in the delay ratio (mean $R^{2}=.588$ and .606 , respectively). For both rat strains, Block 1 of the descending condition shows preference for the SS lever across delay ratios, resulting in aberrant fits $\left(R^{2}=-.129\right.$ and $-.221)$ and poor estimates of sensitivity $(s=-.069$ and .027 , respectively). Block 2 shows a positive relation between response ratios and delay ratios, with the LEWs showing higher sensitivity to immediacy to reinforcement $(s=.206)$ than the F344s $(s=.055)$. The goodness of fit for the group data of the LEWs in Block $2\left(R^{2}=.943\right)$ suggested that this strain recovered the pattern of choice shown in the ascending condition faster than the F344s $\left(R^{2}=-.042\right)$. For the LEWs, Blocks 3-7 show results consistent with those of the ascending condition; indifference occurred when the LLR was delayed $10 \mathrm{~s}$, preference for the SS lever when it was delayed $20 \mathrm{~s}$ or longer, and preference for the LL lever when the LLR was delayed either 0.1 or $5 \mathrm{~s}$. In contrast, the F344s show a strong preference for the SS lever across delay ratios. The F344s showed a preference for the LL lever when it delivered the LLR with a 0.1-s delay (delay ratio $=0$ ). Note that, as can be seen in Fig. 3, most data points of the LEWs are above those of the F344s, indicating than the LEWs responded more to the LL lever than the F344s did across delay ratios. Yet, estimates of sensitivity to immediacy of reinforcement, ranging from -.069 to .500 for the data of the LEWs $(M=.307)$, were not significantly different $(W=14$, $p>.05$ ) from those for the data of the F344s, ranging from .027 to $.513(M=.315)$.

Following Fig. 3, Blocks 1 and 2 of the random condition show similar choices in both strains; preference for responding on the SS lever is evident across delay ratios. In Blocks 3-7, the F344s emitted more responses on the LL lever than did the LEWs with delays to LLR occurring in random order; note that across delays, most of the data points of the F344s are close to the indifference line. The LEWs show a slight preference for responding on the SS lever across delay ratios, with indifference occurring with delays to LLR of 5 and $10 \mathrm{~s}$ (delay ratios of -5.64 and -6.64 , respectively). For both rat strains, a clear preference for the LL lever occurred when the LLR was delayed $0.1 \mathrm{~s}$ (delay ratio $=0$ ). With delays to LLR of 5 and $10 \mathrm{~s}$, the F344s showed preference for the LL lever and the LEWs showed indifference. Again, estimates of sensitivity to the immediacy of reinforcement (see Table 3), ranging from .114 to $.222(M=.171)$ for the data of LEWs, were not significantly different $(W=25, p>.05)$ from those for the F344s, ranging from .105 to $.182(M=.156)$.

In the reascending condition, following the corresponding parameters presented in Table 3, the choices of the LEWs and F344s generated the steepest slopes (mean $s$ s $=.437$ and .344 , respectively), suggesting that sensitivity to the immediacy of reinforcement increased with extensive training in the choice situation. Equation 2 accounted for a good proportion of the variability in the choices of the LEWs and F344s $\left(\right.$ mean $R^{2} \mathrm{~s}=$
.622 and .786, respectively, as is shown in Table 3) that occurred with within-session changes in the delay ratio. Both rat strains showed a bias for responding on the LL lever, with estimates of bias for the data of the LEWs, ranging from 1.428 to $2.563(M=2.126)$, not being significantly different $(W=15, p>.05)$ from those of the $\mathrm{F} 344 \mathrm{~s}$, ranging from 1.891 to $2.341(M=2.144)$.

In Blocks 1 and 2, the F344s emitted more responses on the LL lever that did the LEWs across delay ratios, showing less sensitivity to within-session changes in the immediacy of reinforcement. All blocks show that both strains chose indifferently between LL and SS levers with a 10-s delay to LLR (i.e., -6.64 delay ratio); again, preference for the SS lever occurred when the LLR was delayed $20 \mathrm{~s}$ or longer (delay ratios -7.64 , -8.64, and -9.64), and preference for the LL lever when it delivered the LLR with delays of 5 and $0.1 \mathrm{~s}$ (delay ratios 5.64 and 0 , respectively); note that in Blocks 3-7, most data points of the LEWs overlap with those of the F344s, suggesting similar choices across delay ratios. Estimates of sensitivity for the data of the LEWs, however, ranging from .261 to .574 $(M=.437)$, were significantly higher $(W=27, p<.05)$ than those for the $\mathrm{F} 344 \mathrm{~s}$, ranging from .274 to $.447(M=.344)$, showing in the former strain more sensitivity to changes in the immediacy of reinforcement.

Figure 4 shows food-by-food sensitivity of choices to within-session changes in the immediacy of reinforcement. Except for the first food delivery of the random condition, showing for the data of both strains poor estimates of sensitivity ( $s=-.026$ and -.018), all food deliveries across conditions show a positive relation between the log of the response ratio and the log of the delay ratio. Food by food, Eq. 2 provided better fits to the data points of the F344s than to those of the LEWs in the ascending (mean $R^{2}=.708$ vs. .678), descending (mean $R^{2}=.951$ vs. .772), random (mean $R^{2}=$ .882 vs. .654), and reascending (mean $R^{2}=.791$ vs. .623) conditions. Following the parameters presented in Table 4, both rat strains developed a bias for responding on the LL lever; in the ascending condition and its replication, estimates of bias for the group data of the LEWs, ranging from 1.321 to $1.532(M=1.415)$ and from 1.635 to $2.417(M=2.221)$, respectively, were not significantly different $(W=14, p>$ .05 , and $W=35, p>.05$, respectively) from those for the group data of the F344s, ranging from 1.166 to $1.742(M=$ $1.479)$ and from 1.254 to $2.754(M=2.182)$, respectively. However, the descending and random conditions generated estimates of bias for the data of the LEWs, ranging from 0.424 to $1.297(M=1.043)$ and from -0.973 to $1.304(M=$ $0.798)$, respectively, that were significantly different $(W=53$, $p<.05$, and $\mathrm{W}=10, p<.05$ ) from those corresponding to the F344s, ranging from -0.688 to $1.365(M=0.535)$ and from 0.001 to $1.454(M=1.100)$, respectively.

Sensitivity of preference to within-session changes in the delay ratio increased with each consecutive food delivery, 

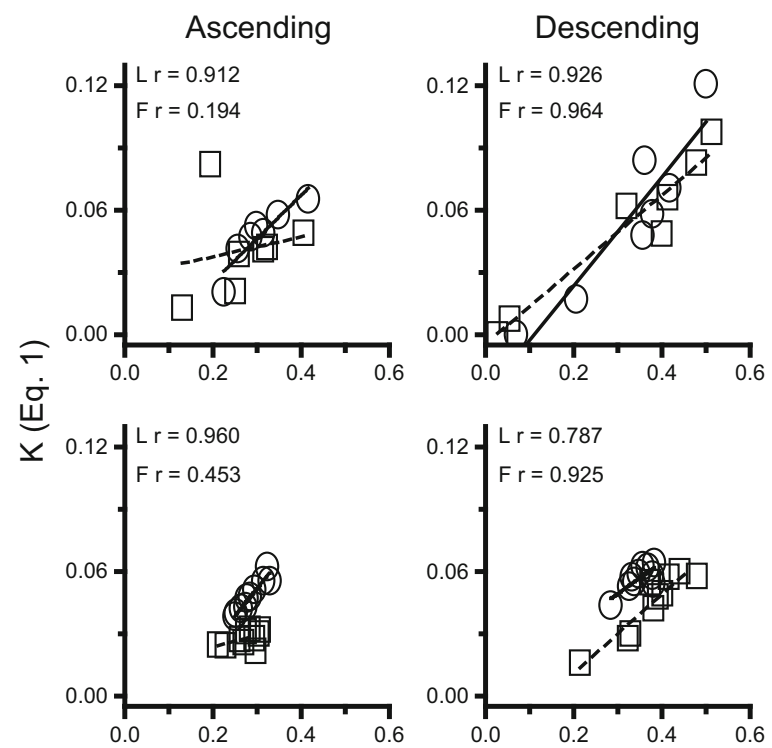

\section{Slope}

Fig. 5 Values of $k$ (Eq. 1) against values of slope ( $s$ in Eq. 2), obtained across blocks of sessions (top panels) and food deliveries (bottom panels). The panels show values obtained for the ascending, descending, random,

with both strains showing similar patterns of choice. Food deliveries of the ascending condition show that the LEWs and F344s preferred the LL lever when the LLR was delayed 0.1 or $5 \mathrm{~s}$ in that lever, indifference when the LLR was delayed $10 \mathrm{~s}$, and preference for the SS lever when the LLR was delayed 20 or more seconds (delay ratios of $-7.64,-8.64$, and -9.64). Estimates of sensitivity for the data of the LEWs, ranging from .249 to $.328(M=.286)$ were not significantly different $(W=42, p>.05)$ from those corresponding to the F344s, ranging from .212 to $.307(M=.273)$.

The descending condition shows, food by food, that the F344s preferred the SS lever when the log delay ratios were different from zero. When the SS and LL levers delivered the LLR with the same 0.1 -s delay (i.e., delay ratio $=0$ ), the F344s preferred the LL lever. Food by food, the LEWs also preferred the LL lever when both levers delivered the LLR with the same 0.1 -s delay (i.e., delay ratio $=0$ ), but with delay ratios of -5.64 and -6.64 , the LEWs showed indifference, and a preference for the SS lever with delay ratios of $-7.64,-8.64$, and -9.64 . Estimates of sensitivity for the data of the LEWs, ranging from .285 to $.381(M=.350)$, were not significantly different $(W=18, p>.05)$ from those for the data of the F344s, ranging from .214 to $.480(M=.375)$.

Food Deliveries 1-2 of the random condition show data points of the LEWs and F344s that fall on the indifference line across delay ratios, suggesting a lack of sensitivity of the behavior ratio to within-session changes in delay ratio $(s=-.026$ and .063 vs. -.018 and .059 , respectively). Sensitivity to the immediacy of reinforcement gradually emerged with Food Deliveries 3-10 of the random condition, with the F344s emitting more responses on the LL lever than did the LEWs across
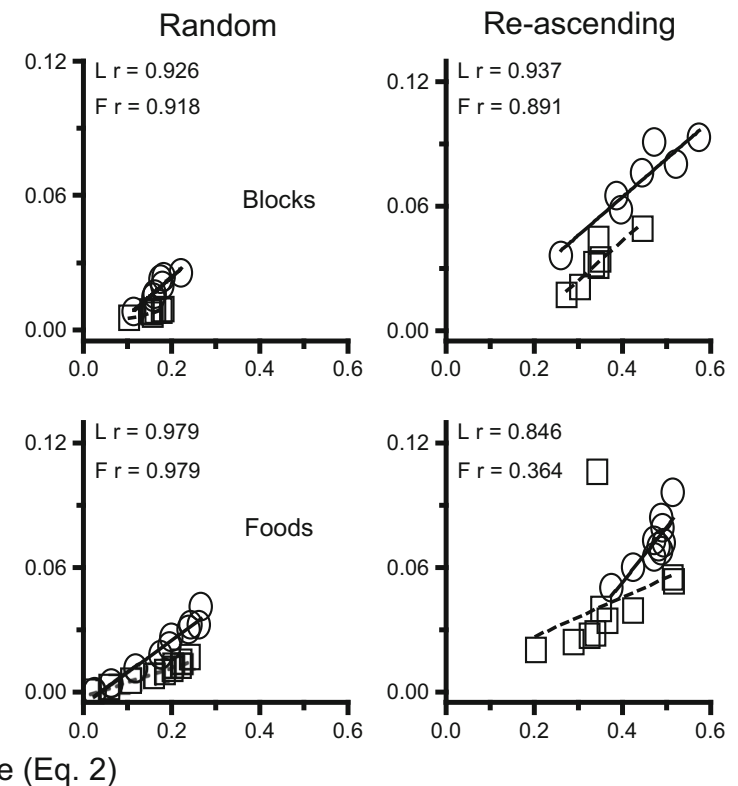

and redetermination of the ascending delay-order condition. Pearson's $r$ values, computed using the means of the groups (LEW in circles and F344 in squares), appear near the data points

delay ratios. Estimates of sensitivity for the data of the LEWs, ranging from -.026 to $.267(M=.174)$, were significantly higher $(W=51, p<.05)$ than those of the F344 rats, ranging from -.018 to $.242(M=.159)$. Both strains preferred the SS lever when the LLR was delayed 40 or $80 \mathrm{~s}$; indifference occurred when the LLR was delayed 20,10, or $5 \mathrm{~s}$; and preference for the LL lever when the LLR was delivered in both levers within a 0.1 -s delay.

Again, the reascending condition shows the steepest slopes, with estimates of sensitivity for the data of the LEWs ranging from .376 to $.515(M=.471)$ that are significantly higher $(W=$ $52, p<.05)$ than those for the F344s, ranging from .205 to .517 $(M=.368)$. The pattern of choices that both strains established in the ascending condition was recovered in the replication of this condition. All food deliveries show preference for the LL lever when it delivered the LLR with delays of 0.1 and $5 \mathrm{~s}$, indifference with a 10-s delay, and preference for the SS lever when the LL lever delayed the LLR by 20 or more seconds (i.e., for delay ratios of $-7.64,-8.64$, and -9.64 ).

To summarize, the analysis using the generalized matching law confirmed what had previously been found using the hyperbolic-decay function; there were no consistent differences in impulsive choices between strains during the ascending presentation-order-of-delays condition. During the descending condition, the LEWs recovered the pattern of choice established in the ascending condition faster than the F344s, and showed higher sensitivity to the immediacy of reinforcement. In the random condition, both strains preferred the LL lever when the delay to LLR was $0.1 \mathrm{~s}$; with longer delays, the F344s maintained this preference for the LL lever, whereas LEWs shifted to indifference. The reascending condition 

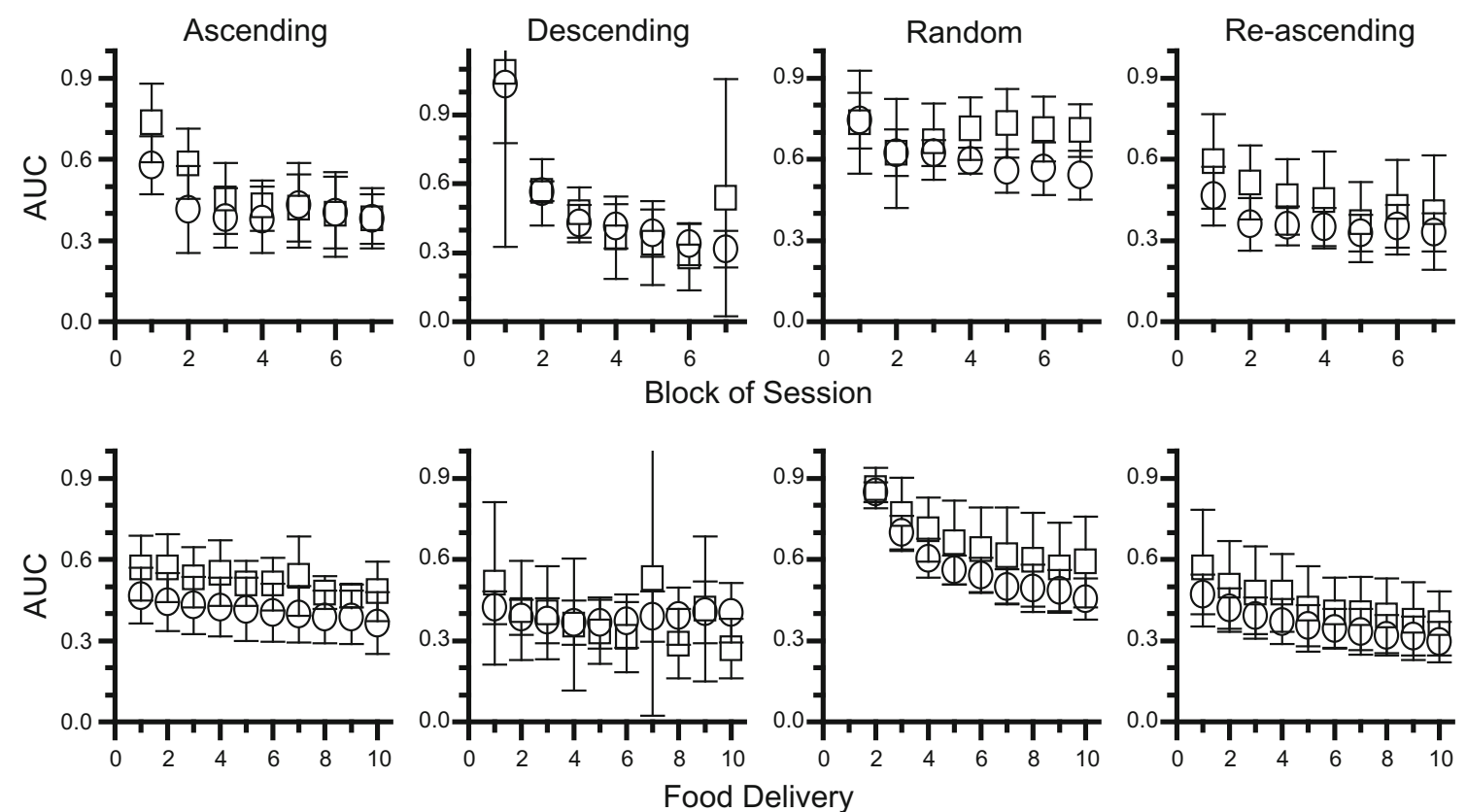

Fig. 6 Values of area under the curve (AUC) as a function of block of sessions (top panels) and food deliveries (bottom panels). Other details are as in Fig. 1

showed that sensitivity to the immediacy of reinforcement increased as a function of training, with the LEWs showing higher sensitivity to immediacy than did the F344s.

\section{Hyperbolic-decay function and the general form of the matching law}

The results in Figs. 1, 2, 3 and 4 suggest some regularity between Eqs. 1 and 2: Both models of choice provided good fits to the data of both strains, with the former generating estimates of discounting rate $(k)$ and the latter generating estimates of sensitivity to the immediacy of reinforcement $(s)$ that might be positively correlated to one another (e.g., Aparicio et al., 2013). To explore this possibility, estimates of $k$ (Eq. 1) are plotted in Fig. 5 against estimates of $s$ (Eq. 2), computed for blocks of sessions (upper graphs) and food deliveries (lower graphs) of the ascending, descending, random, and replication to ascending presentation-order-of-delays conditions. The continuous and dashed lines are the best fits using linear regression; resulting values of Pearson's $r$ appear in the left corner of each graph for the data corresponding to the LEWs $(\mathrm{L} r$ ) and F344s (F $r$ ).

All graphs in Fig. 5 show a positive correlation between estimates of $k$ and estimates of $s$. For blocks of sessions of the ascending condition, the regression lines show a higher correlation for the group data of the LEWs $(r=.912)$ than for the group data of the F344s $(r=.194)$, resulting in a better fit to estimates of $k$ and $s\left(R^{2} \mathrm{~s}=.799\right.$ and -.155 , respectively). Regression lines were fitted to the data that individual LEWs and F344s generated in blocks of sessions (graphs not shown). The resulting Pearson's $r$ s, ranging from .539 to $.988(M=$
.989) for the LEWs, were not significantly different $(U=43$, $p>.05)$ from the corresponding Pearson's $r$ s for the F344s, ranging from .747 to $.926(M=.860)$. For food deliveries, the regression lines show a higher correlation $(r=.960)$ and better fit $\left(R^{2}=.913\right)$ for the group data of the LEWs than for the group data of the F344s $\left(r=.453, R^{2}=.106\right)$. Regression lines were also fitted to the data that individual LEW and F344 rats generated for each food delivery (graphs not shown). The resulting Pearson's $r \mathrm{~s}$, ranging from .106 to $.798(M=.295)$ for the data of the individual LEWs, were not significantly different $(U=29, p>.05)$ from those of the F344s, ranging from -.208 to $.981(M=.620)$.

For both rat strains, blocks of sessions of the descending condition show parallel correlations ( $r \mathrm{~s}=.926$ and .964 , respectively), but the fit to the data of the F344s was better $\left(R^{2}=.916\right)$ than that to the data of the LEWs $\left(R^{2}=.830\right)$. Pearson's $r \mathrm{~s}$, ranging from -.101 to $.562(M=.215)$ for correlations with the data of the individual LEWs, were not significantly different $(U=23, p>.05)$ from those correlations with the data of the individual $\mathrm{F} 344 \mathrm{~s}$, ranging from -.262 to $.781(M=.340)$. Consistent with the results of blocks of sessions, food deliveries showed a higher correlation $(r=.925)$ and better fit $\left(R^{2}=.840\right)$ for the data of the F344s than for the data of the LEWs $(r=.787$, $\left.R^{2}=.572\right)$. Pearson's $r$ s, ranging from -.223 to $.959(M=.340)$ for the data of the individual LEWs, were not significantly different $(U=23, p>.05)$ from those for the data of individual $\mathrm{F} 344 \mathrm{~s}$, ranging from -.754 to $.944(M=.558)$.

The random condition shows data points that are clustered near the origin, due to low estimates of $k$ and $s$, with similar correlations $(r=.926$ and .918$)$ and goodnesses of fit $\left(R^{2}=\right.$ .818 and .754) for the data of the LEWs and F344s, 
respectively. Pearson's $r$ s, ranging from .890 to $.993(M=$ .947) for the data of the individual LEWs, were significantly higher $(U=64, p<.05)$ from those corresponding to the data of the individual F344s, ranging from .065 to $.825(M=.468)$. Food deliveries show a remarkable result: The correlation and goodness of fit for the data of the LEWs $\left(r=.979\right.$ and $R^{2}=$ $.954)$ are identical to those computed for the F344s. Pearson's $r$ s, ranging from .928 to $.976(M=.949)$ for the data of the individual LEWs, were not significantly different $(U=43, p>$ .05 ) from those for the data of the individual F344s, ranging from .863 to $.973(M=.931)$.

For the data of the LEWs and F344s, blocks of sessions of the replication of the ascending condition show positive correlations $(r=.937$ and .891$)$ and good fits $\left(R^{2}=.854\right.$ and .754 , respectively). Pearson's $r$ s, ranging from .320 to .958 ( $M=$ .708), for the data of the individual LEWs were significantly different $(U=12, p<.05)$ from those for the data of the individual F344s, ranging from .855 to $.957(M=.922)$. Food deliveries show that the correlation for the data of the LEWs was higher $(r=.846)$ than that for the data of the F344s $(r=$ $.364)$, resulting in a better fit to estimates of $k$ and $s\left(R^{2} \mathrm{~s}=.681\right.$ and .024 , respectively). Pearson's $r \mathrm{~s}$, ranging from -.293 to $.880(M=.653)$, for the data of the individual LEWs were not significantly different $(U=13, p>.05)$ from those for the data of the individual F344s, ranging from -.493 to $.959(M=.686)$.

\section{Area under the curve}

Computations of the AUC (Myerson et al., 2001) for the group data of the LEWs (circles) and F344s (squares) are plotted in Fig. 6 as a function of blocks of sessions (upper graphs) and food deliveries (lower graphs).

Blocks 1 and 2 of the ascending condition show that at the beginning of the study, the LEWs discounted the LLR more than the F344s, but that between-strain difference in discounting the LLR decreased in Blocks 3-4 and disappeared in Blocks 5-7, showing similar discountings of the LLR for both rat strains. Estimates of the AUC, ranging from .379 to $.577(M=.423)$, for the data of the LEWs were not significantly different $(W=5, p>.05)$ from those for the data of the F344s, ranging from .381 to $.734(M=.485)$. Food by food, both rat strains show moderate discounting of the LLR, with the F344s discounting the LLR less than the LEWs across food deliveries; estimates of the AUC for the LEWs, ranging from .364 to $.466(M=.411)$, were significantly lower $(W=0, p<$ $.05)$ than those for the F344s, ranging .464 to $.568(M=.520)$.

For both rat strains, the descending condition shows that proportions decreased with increasing blocks of sessions, with Block 1 showing no discounting, and Block 7 showing the maximum discounting reached (.300) in that condition (but see the data for the F344s). Estimates of the AUC for the data of the LEWs, ranging from .315 to $1.00(M=.497)$, were not significantly different $(W=12, p>.05)$ from those for the data of the F344s, ranging from .281 to $1.00(M=.522)$. Food deliveries of the descending condition show that, food by food, the LEWs and F344s discounted the LLR with similar proportions (about .350). Estimates of the AUC for the data of the LEWs, ranging from .365 to $.421(M=.387)$, were not significantly different $(W=29, p<.05)$ from those for the data of the F344 rats, ranging from .270 to $.530(M=.383)$.

In the random condition, the LEWs and F344s show the highest proportions (about .650) across blocks of sessions, indicating little discounting of the LLR with delays to its delivery occurring within sessions in random order. Blocks 5-7, however, show that the LEWs discounted the LLR more than the F344s (AUCs $=.500$ vs. .650 , respectively). Yet, estimates of the AUC for the data of the LEWs, ranging from .541 to $.742(M=.607)$, were not significantly different $(W=3, p>.05)$ from those for the data of the F344s, ranging from .621 to $.736(M=.698)$. Food deliveries show that the AUC decreased with each food delivery of the random condition, with the LEWs showing a decrease in AUC values from .850 with the first food delivery to about .400 with the last food delivery, and the F344s also showing a decrease, from .850 with the first food delivery to about .600 with the last food delivery. Estimates of the AUC for the LEWs, ranging from .453 to $1.00(M=.628)$ were significantly lower $(W=2, p<.05)$ than those for the F344 rats, ranging from .568 to $1.00(M=.707)$.

The reascending condition generated estimates of the AUC that were comparable to those computed for the first determination of this condition, with the LEWs discounting the LLR more than the F344s across blocks of sessions. Estimates of the AUC for the data of the LEWs, ranging from .327 to .464 ( $M=$ $.362)$, were significantly lower $(W=0, p<.05)$ than those for the data of the F344 rats, ranging from .367 to $.590(M=.458)$. Food deliveries of the reascending condition show similar estimates of the AUCs for the data of the LEWs and F344s across food deliveries, with the LEWs showing more discounting of the LLR than the F344s show, food by food. Estimates of the AUC for the LEWs, ranging from .294 to $.470(M=.361)$, were significantly lower $(W=0, p<.05)$ than those for the data of the F344s, ranging .363 to $.568(M=.437)$.

In sum, the AUC analysis confirmed that strain differences were not consistent throughout the seven blocks of sessions and food deliveries of the ascending condition; only during the first two blocks of training did the LEWs show steeper discounting. The variability of AUC measures within strains increased the most when the presentation order of delays to LLR changed from ascending to descending; this is additional evidence supporting the disruptive effect of the descending presentation order of delays to LLR. In the random condition, both strains showed the highest AUCs across blocks of sessions and food deliveries, showing no consistent differences in impulsive choices between strains. The results from the two first blocks of the ascending condition were replicated in the 
reascending condition, with the LEWs showing more discounting than the F344s.

\section{Discussion}

The present study has clearly showed that impulsive choice can change as a function of learning and is not a static property of behavior that is mainly determined by genetic and neurochemical mechanisms; thus, when examining impulsive choice, we deal with a dynamic system. We contend that a working definition of impulsivity is a complicated task and that the evidence from animal models to examine impulsivity is warranted by studies that have incorporated novel procedures and several ways to estimate impulsive choice. Accordingly, we developed a concurrent-chains procedure in which response allocation in two levers concurrently available in the initial link was used to assess preference (Grace, 1994). One terminal link varied the delay to the LLR within sessions, capitalizing on Evenden and Ryan's (1996) method for generating an entire delay-of-reinforcement function within each session. Because more than one response on the same lever was allowed to choose between the SSR and the LLR, the probability to develop an exclusive preference for one or the other lever was reduced; this is important, because exclusive preference occurs when a single response serves to choose and produce either the SSR or the LLR (Mazur, 1987, 2010). Our procedure required locomotion to travel from the front to the back wall of the chamber and press on a lever there to start each cycle of the concurrent-chains schedule; these activities implied some effort representing a cost to reach the choice link; effort plays an important role in preference (Aparicio, 2001; Aparicio \& Cabrera, 2001; Aparicio \& Otero, 2004; Salamone \& Correa, 2009), and cost influences delay discounting (Paglieri, 2013). We also implemented multiple analytical tools, providing an ideal scenario to generate other critical measures and further examine strain differences in impulsive choice.

The study of impulsivity tends to be predominantly linked to pathologies, maladaptive behavior, and personality (Odum, 2011), when in actuality it is a pattern of behavior that may also be adaptive in humans and nonhuman animals (Fawcett, McNamara, \& Houston, 2012). In the present study, we applied a behavioral analytical approach, defined impulsivity functionally rather than structurally, and provided a better understanding of such a concept by keeping the definition of impulsivity open to empirical evidence (Winstanley, Eagle, \& Robbins, 2006).

Historical variables are worth further exploring within the context of impulsivity. In the present study, LEWs and F344s were exposed to the same sequences of ascending, descending, random, and reascending presentation orders of delays to LLR; hence, varying the order in which this sequence of conditions was presented is a question that deserves further examination. Also, we studied the impulsive choices of the LEWs and F344s for most of their life span, and aging might have contributed to the result showing that the F344s chose the LL lever at higher proportions than did the LEWs during the random and the replication of the ascending presentationorder-of-delays conditions; this finding resembles that obtained with aged F344 rats that, regardless of their experience with the choice situation, preferred the LLR over the SSR (Simon et al., 2010). Nonetheless, we did not examine in isolation the role of aging in determining the preference for the LLR. Still, though, as in Aparicio et al. (2013), the results confirmed that the impulsive choices of LEWs and F344s changed with learning; early in training, the LEWs showed more impulsive choices (as estimated by $k$ ) than the F344s, but this betweenstrain difference in impulsive choices disappeared with experience in the choice situation, showing parallel discounting functions (similar estimates of $k$ for the LEWs and F344s) in the last blocks of sessions of the ascending condition. This finding calls for a reevaluation of studies that have exposed these rat strains to procedures designed to examine impulsivity for shorter lengths of time (Stein et al., 2012; Wilhelm \& Mitchell, 2009).

On the basis of the present and previous discoveries (Aparicio et al., 2013), it is fair to conclude that the behavior pattern labeled impulsivity, frequently implicated in maladaptive behavior, can actually change as a function of learning. Additional evidence of the effect of learning on preference was observed in the descending condition; presenting the delays to LLR in descending order disrupted the patterns of choice that the LEWs and F344s established responding to delays to LLR in ascending order. It could be said that in adjusting to the transition from the ascending to the descending presentation order of delays, the LEWs did so more rapidly than the F344s, choosing the LL lever at higher proportions during most delays to LLR. This result is in dispute with previous findings (Anderson \& Diller, 2010; Anderson \& Woolverton, 2005; Huskinson et al., 2012; Madden et al., 2008; Stein et al., 2012) that showed more impulsivity in the LEWs; the assertion that the LEWs choose more impulsively than the F344s is not supported by the present study, challenging this strain difference and confirming that it may be due to procedural issues (Stein et al., 2012) and the analytical tools used to characterize impulsive choice (Madden \& Johnson, 2010).

We conclude that strain differences in impulsivity are not fundamentally a direct function of their neurochemical characteristics (Burnet, Mefford, Smith, Gold, \& Sternberg, 1992; Chaouloff et al., 1995; Flores et al., 1998; Lindley et al., 1999; Sziraki et al., 2001), but as we have shown, differences in impulsive choice between LEWs and F344s change as a function of learning (Aparicio et al., 2013). Overall, the discounting functions of the LEWs across delay conditions 
seem to suggest that they are more adaptable to dynamic changes in the contingencies of reinforcement than are F344s. Particularly, the present results seem to indicate that the LEWs are more sensitive to rapid changes in delay to reinforcement, in the sense that their preferences changed sooner than those of the F344s. It could be concluded that impulsivity is an advantage in dynamic changing environments that might be adaptive for human and nonhuman animals (Fawcett et al., 2012).

The present study extended the generality of the hyperbolic-decay model (Mazur, 1987) and the general form of the matching law (Baum, 1974; Baum \& Rachlin, 1969) to the study of impulsive choice. The former model has been successful in describing the data from several experiments on delay discounting in which human and nonhuman animals have been exposed to a variety of procedures examining impulsivity (e.g., Mazur, 2000; Richards et al., 1997; Stein et al., 2012); in agreement with these outcomes, the present results showed that delay discounting in LEWs and F344s was well described by Mazur's (1987) hyperbolic-decay model. This result, first reported by Stein et al. (2012), using a rapid version of a steady-state adjusting-amount procedure developed by Mazur (2000) and adapted from Richards et al.'s (1997) method, was also documented in a study using a concurrentchains procedure similar to that used here (Aparicio et al., 2013). Although the general form of the matching law (Baum, 1974; Baum \& Rachlin, 1969) has not been extensively used to analyze impulsive choice (e.g., Ainslie, 1992; Aparicio et al., 2013), it accurately described the data from the present study. Consistent with estimates of $k$ obtained with Eq. 1, estimates of $s$ using Eq. 2 also changed across conditions, with the random presentation-order-of-delays condition showing the lowest estimates of $s$ and the redetermination to the ascending condition the highest estimates, suggesting that both the presentation order of the delays to the LLR and the length of training are important factors in determining the sensitivity of preference to dynamic changes in delay to reinforcement (Aparicio et al., 2013).

We demonstrated that using both the hyperbolic-decay model and the general form of the matching law in conjunction to describe impulsive choice provided the possibility of observing compatibility between models of choice, which in turn might offer different ways to analyze impulsivity in nonhuman animals (Aparicio et al., 2013); we observed prevalent high and positive correlations between estimates of discounting rates ( $k$ in Eq. 1$)$ and estimates of sensitivity ( $s$ in Eq. 2) across blocks of sessions and food deliveries, confirming that the matching law (Herrnstein, 1970) accurately describes impulsive choice in concurrent-chains procedures (Aparicio et al., 2013).

The analyses of the AUC using Myerson et al.'s (2001) method confirmed results showing that between-rat-strain differences in preference changed as a function of the presentation order of delays to LLR and experience in the choice situation. The analysis of blocks of sessions showed no clear between-strain differences in the AUC. For both rat strains, the AUC decreased with progressive training and consecutive food deliveries within delay components. In the descending condition, the AUC was higher for the LEWs across blocks of sessions and food deliveries, but the random condition showed the opposite result: The AUC was higher for the F344s than for the LEWs, and this result was confirmed in the redetermination of the ascending presentation order of delays. Interestingly, for both rat strains the analysis across food deliveries revealed values of the AUC that gradually decreased with consecutive food deliveries, showing a gradual shift from self-controlled choice to impulsive choice with extended training (Aparicio et al., 2013).

An important aspect of the present study was using FT instead of FI schedules in the terminal links of the concurrent-chains procedure to remove the response producing the LLR. Accordingly, we analyzed the distribution of responses during the FT terminal link (computations not shown) to assess the possibility of an accidental reinforcement at the end of the FT schedule (Skinner, 1948). Responses during delays of $5,10,20,40$, and $80 \mathrm{~s}$ to LLR were counted and sorted in bins of $1 \mathrm{~s}$ each across sessions of the same condition. These computations revealed that FT responses decreased with increasing delays to the LLR; the LEWs and F344s emitted more responses at the start of each delay, showing no evidence of the scalloped pattern of responding characterizing FI schedules and discarding the possibility of fortuitous reinforcement at the end of the FT (Skinner, 1948). This finding is consistent with the idea that nonhuman animals are indifferent to FI and FT terminal links of concurrent-chains schedules (Neuringer, 1969).

In conclusion, through the present study we investigated both the acquisition and maintenance of the impulsive behavior of LEWs and F344s, and found more dynamic changes in choice characterizing impulsivity than those previously reported in studies using a rapid-redetermination assessment of indifference points (e.g., Stein et al., 2012; Wilhelm \& Mitchell, 2009) and steady-state procedures (e.g., Anderson \& Diller, 2010; Anderson \& Woolverton, 2005; Huskinson et al., 2012; Madden et al., 2008; Stein et al., 2012). Consistent with findings obtained in nonhuman (Fox et al., 2008) and human (Robles \& Vargas, 2007, 2008; Robles et al., 2009) animals, we found that the presentation order of delays to the LLR is important in determining the discounting rate ( $k$ in Eq. 1) and sensitivity to the immediacy of reinforcement ( $s$ in Eq. 2). We found that the descending presentation order of delay to LLR disrupted the pattern of choices that LEWs and F344s established responding to delays to LLR in the ascending and random conditions. Our results supported the notion that strain differences in impulsivity change as a function of training (Aparicio et al., 2013). 
Differences in impulsive choice between LEWs and F344s should be studied carefully and for longer periods of time, because they are not static properties of behavior solely determined by genetic and neurochemical brain mechanisms. We concluded that LEWs are more adaptable to dynamic changes in the contingencies of reinforcement than are F344s, and that impulsivity is an advantage in dynamic reinforcing environments that might be adaptive for both human and nonhuman animals (Fawcett et al., 2012). As has recently been noted, concurrent-chains procedures have distinct advantages over the techniques typically used to study delay discounting, and they can be used without worrying that new discoveries can be accredited to the use of innovative techniques (Oliveira, Green, \& Myerson, 2014).

Author Note Portions of this article were presented at the 38th and 39th Annual Conventions of the Association for Behavior Analysis International.

\section{References}

Ainslie, G. W. (1974). Impulsive control in pigeons. Journal of the Experimental Analysis of Behavior, 21, 485-489. doi:10.1901/ jeab. 1974.21-485

Ainslie, G. W. (1992). Picoeconomics: The strategic interaction of successive motivational states within the person. New York, NY: Cambridge University Press.

Alsop, B., \& Davison, M. (1986). Preference for multiple versus mixed schedules of reinforcement. Journal of the Experimental Analysis of Behavior, 45, 33-45. doi:10.1901/jeab.1986.45.33

Anderson, K. G., \& Diller, J. W. (2010). Effects of acute and repeated nicotine administration on delay discounting in Lewis and Fischer 344 rats. Behavioural Pharmacology, 21, 754-764. doi:10.1097/ FBP.0b013e328340a050

Anderson, K. G., \& Elcoro, M. (2007). Response acquisition with delayed reinforcement in Lewis and Fischer 344 rats. Behavioural Processes, 74, 311-318. doi:10.1016/j.beproc.2006.11.006

Anderson, K. G., \& Woolverton, W. L. (2005). Effects of clomipramine on self-control choices in Lewis and Fischer 344 rats. Pharmacology, Biochemistry and Behavior, 80, 387-393. doi:10. 1016/j.pbb.2004.11.015

Aparicio, C. F. (2001). Overmatching in rats: The barrier choice paradigm. Journal of the Experimental Analysis of Behavior, 75, 93106. doi:10.1901/jeab. 2001.75-93

Aparicio, C. F., \& Cabrera, F. (2001). Choice with multiple alternatives: The barrier choice paradigm. Mexican Journal of Behavior Analysis, 27, 97-118.

Aparicio, C. F., Hughes, C. E., \& Pitts, R. C. (2013). Impulsive choice in Lewis and Fischer 344 rats: Effects of extended training. Conductual, 1(3), 22-46. Retrieved from http://conductual.com/ content/impulsive-choice-lewis-and-fischer-344-rats

Aparicio, C. F., \& Otero, E. (2004). Sensitivity to reinforcement and changeover requirements in dynamic and quasi-stable environments. Mexican Journal of Behavior Analysis, 30, 23-78.

Baum, W. M. (1974). On two types of deviations from the matching law: Bias and undermatching. Journal of the Experimental Analysis of Behavior, 22, 231-242. doi:10.1901/jeab. 1974.22-231

Baum, W. M., \& Rachlin, H. C. (1969). Choice as time allocation. Journal of the Experimental Analysis of Behavior, 12, 861-874. doi:10.1901/jeab. 1969.12-861
Brower, V. G., Fu, Y., Matta, S. G., \& Sharp, B. M. (2002). Rat strain differences in nicotine self-administration using an unlimited access paradigm. Brain Research, 930, 12-20. doi:10.1016/S00068993(01)03375-3

Brown, P. L., \& Jenkins, H. M. (1968). Auto-shaping of the pigeon's keypeck. Journal of the Experimental Analysis of Behavior, 11, 1-8. doi:10.1901/jeab. 1968.11-1

Burnet, P. W., Mefford, I. N., Smith, C. C., Gold, P. W., \& Sternberg, E. M. (1992). Hippocampal 8-[3H]Hydroxy-2-(Di-n-Propylamino) tetralin binding site densities, serotonin receptor $\left(5-\mathrm{HT}_{1 \mathrm{~A}}\right)$ messenger ribonucleic acid abundance, and serotonin levels parallel the activity of the hypothalamopituitary-adrenal axis in rat. Journal of Neurochemistry, 59, 1062-1070.

Burnet, P. W., Mefford, I. N., Smith, C. C., Gold, P. W., \& Sternberg, E. M. (1996). Hippocampal 5-HT1A receptor binding sites densities, 5-HT1A receptor messenger ribonucleic acid abundance and serotonin levels parallel the activity of the hypothalamo-pituitary-adrenal axis in rats. Behavioural Brain Research, 73, 365-368. doi:10. 1016/0166-4328(96)00116-7

Chaouloff, F., Kulikov, A., Sarrieau, A., Castanon, N., \& Mormede, P. (1995). Male Fischer 344 and Lewis rats display differences in locomotor reactivity, but not in anxiety-related behaviours: Relationship with the hippocampal serotonergic system. Brain Research, 693, 169-178. doi:10.1016/0006-8993(95)00733-7

Evenden, J. L., \& Ryan, C. N. (1996). The pharmacology of impulsive behavior in rats: The effects of drugs on response choice varying delays of reinforcement. Psychopharmacology, 128, 161-170. doi: 10.1007/s002130050121

Evenden, J. L., \& Ryan, C. N. (1999). The pharmacology of impulsive behavior in rats VI: The effects of ethanol and selective serotonergic drugs on response choice with varying delays of reinforcement. Psychopharmacology, 146, 413-421. doi:10.1007/PL00005486

Farrar, A. M., Kieres, A. K., Hausknecht, K. A., de Wit, H., \& Richards, J. B. (2003). Effects of reinforcer magnitude on an animal model of impulsive behavior. Behavioural Processes, 64, 261-271. doi:10. 1016/S0376-6357(03)00139-6

Fawcett, T. W., McNamara, J. M., \& Houston, A. I. (2012). When is it adaptive to be patient? A general framework for evaluating delayed rewards. Behavioural Processes, 89, 128-136. doi:10.1016/j. beproc.2011.08.015

Flores, G., Wood, G. K., Barbeau, D., Quiron, R., \& Srivastava, L. K. (1998). Lewis and Fischer 344 rats: A comparison of dopamine transporter and receptors. Brain Research, 814, 34-40. doi:10. 1016/S0006-8993(98)01011-7

Fox, A. T., Hand, D. J., \& Reilly, M. P. (2008). Impulsive choice in a rodent model of attention-deficit/hyperactivity disorder. Behavioural Brain Research, 187, 146-152. doi:10.1016/j.bbr. 2007.09.008

Garcia-Lecumberri, C., Torres, I., Martin, S., Crespo, J. A., Miguens, M., Nicanor, C., \& Ambrosio, E. (2011). Strain differences in the doseresponse relationship for morphine self-administration and impulsive choice between Lewis and Fischer 344 rats. Journal of Psychopharmacology, 25, 783-791. doi:10.1177/ 0269881110367444

Grace, R. C. (1994). A contextual model of concurrent-chains choice. Journal of the Experimental Analysis of Behavior, 61, 113-129. doi:10.1901/jeab. 1994.61-113

Green, L., Myerson, J., Shah, A. K., Estle, S. J., \& Holt, D. D. (2007). Do adjusting-amount and adjusting-delay procedures produce equivalent estimates of subjective value in pigeons? Journal of the Experimental Analysis of Behavior, 87, 337-347. doi:10.1901/ jeab. 2007.37-06

Herrnstein, R. J. (1970). On the law of effect. Journal of the Experimental Analysis of Behavior, 13, 243-266. doi:10.1901/jeab. 1970.13-243

Huskinson, S. L., Krebs, C. A., \& Anderson, K. G. (2012). Strain differences in delay discounting between Lewis and Fischer 344 rats at 
baseline and following acute and chronic administration of d-amphetamine. Pharmacology Biochemistry \& Behavior, 101, 403-416. doi:10.1016/j.bepro.2013.07.013

Kearns, D. N., Gomez-Serrano, M. A., Weiss, S. J., \& Riley, A. L. (2006). A comparison of Lewis and Fischer rat strains on autoshaping (signtracking), discrimination reversal learning and negative auto-maintenance. Behavioral and Brain Research, 169, 193-200. doi:10. 1016/j.bbr.2006.01.005

Kosten, T. A., \& Ambrosio, E. (2002). HPA axis function and drug addictive behaviors: Insights from studies with Lewis and Fischer 344 inbred rats. Psychoneuroendocrinology, 27, 35-69. doi:10. 1016/S0306-4530(01)00035-X

Kosten, T. A., Miserendino, M. J. D., Haile, C. N., DeCaprio, J. L., Jatlow, P. I., \& Nestler, E. J. (1997). Acquisition and maintenance of intravenous cocaine self-administration in Lewis and Fischer inbred rat strains. Brain Research, 778, 418-429. doi:10.1016/S00068993(97)01205-5

Lindley, S. E., Bengoechea, T. G., Wong, D. L., \& Schatzberg, A. F. (1999). Strain differences in mesotelencephalic dopaminergic neuronal regulation between Fischer 344 and Lewis rats. Brain Research, 832, 152-158. doi:10.1016/S0006-8993(99)01446-8

Logue, A. W. (1988). Research on self-control: An integrating framework. Behavioral and Brain Sciences, 11, 665-709. doi:10.1017/ S0140525X00053978

Madden, G. J., \& Johnson, P. S. (2010). A delay-discounting primer. In G. J. Madden \& W. K. Bickel (Eds.), Impulsivity: The behavioral and neurological science of discounting (pp. 1-37). Washington, DC: American Psychological Association.

Madden, G. J., Smith, N. G., Brewer, A. T., Pinkston, J., \& Johnson, P. S. (2008). Steady-state assessment of impulsive choice in Lewis and Fischer 344 rats: Between-condition delay manipulations. Journal of the Experimental Analysis of Behavior, 90, 333-344. doi:10. 1901/jeab. 2008.90-333

Martin, S., Lyupina, Y., Crespo, J. A., Gonzalez, B., Garcia-Lecumberri, C., \& Ambrosio, E. (2003). Genetic differences in NMDA and D1 receptor levels, and operant responding for food and morphine in Lewis and Fischer 344 rats. Brain Research, 973, 205-213. doi:10. 1016/S0006-8993(03)02482-X

Martin, S., Manzanares, J., Corchero, J., Garcia-Lecumberri, C., Crespo, J. A., Fuentes, J. A., \& Ambrosio, E. (1999). Differential basal proenkephalin gene expression in dorsal striatum and nucleus accumbens, and vulnerability to morphine self-administration in Fischer 344 and Lewis rats. Brain Research, 821, 350-355. doi: 10.1016/S0006-8993(99)01122-1

Mazur, J. E. (1987). An adjusting procedure for studying delayed reinforcement. In M. L. Commons, J. E. Mazur, J. A. Nevin, \& H. Rachlin (Eds.), Quantitative analyses of behavior: The effect of delay and of intervening events on reinforcement value (Vol. 5, pp. 5573). Hillsdale, NJ: Erlbaum.

Mazur, J. E. (2000). Tradeoffs among delay, rate and amount of reinforcement. Behavioural Processes, 49, 1-10. doi:10.1016/S0376-6357

Mazur, J. E. (2010). Distributed versus exclusive preference in discretetrial choice. Journal of the Experimental Analysis of Behavior, 36, 321-333. doi:10.1037/a0017588

Mazur, J. E. (2012). Effects of pre-trial response requirements on selfcontrol choices by rats and pigeons. Journal of the Experimental Analysis of Behavior, 97, 215-230. doi:10.1901/jeab. 2012.97-215

Myerson, J., \& Green, L. (1995). Discounting of delayed rewards: Models of individual choice. Journal of the Experimental Analysis of Behavior, 64, 263-276. doi:10.1901/jeab. 1995.64-263

Myerson, J., Green, L., \& Warusawitharana, M. (2001). Area under the curve as a measure of discounting. Journal of the Experimental Analysis of Behavior, 76, 235-243. doi:10.1901/jeab. 1995.64-263

Neuringer, A. J. (1969). Delayed reinforcement versus reinforcement after a fixed interval. Journal of the Experimental Analysis of Behavior, 12, 375-383. doi:10.1901/jeab. 1969.12-375
Odum, A. L. (2011). Delay discounting: I'm a $k$, you're a $k$. Journal of the Experimental Analysis of Behavior, 96, 427-439. doi:10.1901/jeab. 2011.96-423

Oliveira, L., Green, L., \& Myerson, J. (2014). Pigeon's delay discounting functions established using a concurrent-chains procedure. Journal of the Experimental Analysis of Behavior, 102, 151-161. doi:10. $1002 /$ jeab. 97

Paglieri, F. (2013). The cost of delay: Waiting versus postponing in intertemporal choice. Journal of the Experimental Analysis of Behavior, 99, 362-377. doi:10.1002/jeab.18

Pitts, R. C., \& McKinney, A. P. (2005). Effects of methylphenidate and morphine on delay-discounting functions obtained within sessions. Journal of the Experimental Analysis of Behavior, 83, 297-314. doi: 10.1901/jeab. 2005.47-04

Rachlin, H., \& Green, L. (1972). Commitment, choice, and self-control. Journal of the Experimental Analysis of Behavior, 17, 15-22. doi: 10.1901/jeab. 1972.17-15

Rachlin, H., Raineri, A., \& Cross, D. (1991). Subjective probability and delay. Journal of the Experimental Analysis of Behavior, 55, 233244. doi:10.1901/jeab. 1991.55-233

Richards, J. B., Mitchell, S. H., de Wit, H., \& Seiden, L. S. (1997). Determination of discount functions in rats with an adjustingamount procedure. Journal of the Experimental Analysis of Behavior, 67, 353-366. doi:10.1901/jeab. 1997.67-353

Robles, E., \& Vargas, P. A. (2007). Functional parameters of delay discounting assessment tasks: Order of presentation. Behavioural Processes, 75, 237-241. doi:10.1016/j.beproc.2007.02.014

Robles, E., \& Vargas, P. A. (2008). Parameters of delay discounting assessment: Number of trials, effort, and sequential effects. Behavioural Processes, 78, 285-290. doi:10.1016/j.beproc.2007. 10.012

Robles, E., Vargas, P. A., \& Bejarano, R. (2009). Within-subject differences in degree of delay discounting as a function of order of presentation of hypothetical cash rewards. Behavioural Processes, 81, 260-263. doi:10.1016/j.beproc.2009.02.018

Salamone, J. D., \& Correa, M. (2009). Dopamine/adenosine interactions involved in effort-related aspects of food. Appetite, 53, 1-8. doi:10. 1016/j.appet.2009.07.018

Selim, M., \& Bradberry, C. W. (1996). Effect of ethanol on extra cellular 5-HT and glutamate in the nucleus accumbens and prefrontal cortex: Comparison between the Lewis and Fischer 344 rat strains. Brain Research, 716, 157-164. doi:10.1016/0006-8993(95)01385-7

Simon, N. W., LaSarge, C. L., Montgomery, K. S., Williams, M. T., Mendez, I. A., Setlow, B., \& Bizon, J. (2010). Good things come to those who wait: Attenuated discounting of delayed rewards in aged Fischer 344 rats. Neurobiology of Aging, 31, 853-862. doi: 10.1016/j.neurobiolaging.2008.06.004

Skinner, B. F. (1948). "Superstition" in the pigeon. Journal of Experimental Psychology, 38, 168-172. doi:10.1037/h0055873

Slezak, J. M., \& Anderson, K. G. (2009). Effects of variable training, signaled and unsignaled delays, and d-amphetamine on delaydiscounting functions. Behavioural Pharmacology, 20, 424-436. doi:10.1097/FBP.0b013e3283305ef9

Stein, J. S., Pinkston, J. W., Brewer, A. T., Francisco, M. T., \& Madden, G. J. (2012). Delay discounting in Lewis and Fischer 344 rats: Steady-state and rapid-determination adjusting-amount procedures. Journal of the Experimental Analysis of Behavior, 97, 305-321. doi: 10.1901/jeab. 2012.97-305

Stubbs, D. A., \& Pliskoff, S. S. (1969). Concurring responding with fixed relative rate of reinforcement. Journal of the Experimental Analysis of Behavior, 12, 887-895. doi:10.1901/jeab. 1969.12-887

Suzuki, T., George, F. R., \& Meisch, R. A. (1988). Differential establishment and maintenance of oral ethanol reinforced behavior in Lewis and Fischer 344 inbred rats. Journal of Pharmacology and Experimental Therapeutics, 245, 164-170. 
Suzuki, T., George, F. R., \& Meisch, R. A. (1992). Etonitazene delivered orally serves as a reinforcer for Lewis but not Fischer 344 rats. Pharmacology, Biochemistry, \& Behavior, 42, 579-586. doi:10. 1007/s00213-009-1480-0

Sziraki, I., Lipovac, M. N., Hashim, A., Sershen, H., Allen, D., \& Cooper, T. (2001). Differences in nicotine-induced dopamine release and nicotine pharmacokinetics between Lewis and Fischer 344 rats. Neurochemical Research, 26, 609-617. doi:10.1023/ A:1010979018217
Wilhelm, C. J., \& Mitchell, S. H. (2009). Strain differences in delay discounting using inbred rats. Genes, Brain and Behavior, 8, 426434. doi:10.1111/j.1601-183X.2009.00484.x

Winstanley, C. A., Eagle, D. M., \& Robbins, T. W. (2006). Behavioral models of impulsivity in relation to ADHD: Translation between clinical and preclinical studies. Clinical Psychology Review, 26, 379-385. doi:10.1016/j.cpr.2006.01.001 